\title{
Some Recent Results for Nonlinear Acoustics in Combustion Chambers
}

\author{
F. E. C. Culick \\ California Institute of Technology, Pasadena, California 91125
}

\section{Introduction}

C ONDITIONS of high energy densities and low losses in combustion chambers encourage the excitation and sustenance of organized unsteady motions generically called combustion instabilities. The fluctuations, common in propulsion systems, often reach sufficient amplitudes to cause excessive rates of heat transfer to exposed surfaces and unacceptable structural vibrations, causing failure in extreme cases. In many cases, to avoid the occurrence of instabilities, combustion chambers are operated below their maximum performance.

Considerable effort has been spent, for more than four decades, on experimental and analytical programs devoted to solving problems of combustion instabilities. Much of the work has been required to measure quantities which, because of the complex processes involved, cannot be predicted accurately from first principles. Analytical work has been concerned largely with linear behavior, the chief purpose being to predict stability of small disturbances in combustion chambers. Many useful results have been obtained, serving in practice to help design experiments, correlate data, and predict the stability of new systems.

However, linear behavior is only a small part of the general problem. A combustion chamber is an isolated system in so far as its stability is concerned, and unstable disturbances evolve as "self-excited" motions. Hence, their amplitudes will grow indefinitely unless nonlinear processes are effective. Complete understanding of observed behavior will therefore be reached only by treating nonlinear behavior.

In the recent past, increased attention has been paid to nonlinear combustion instabilities. It is particularly important, for practical purposes, to explain the existence of limit cycles and the occurrence of unstable motions in linearly stable systems exposed to large initial disturbances. These matters are far from closed, and although substantial progress has been accomplished, little impact has been made on the development of new systems.

The chief purpose of this paper is to provide a brief review of some recent work on nonlinear acoustics applicable to combustion instabilities, largely in the framework of an approximate analysis. Some connections will be made with the modern theory of nonlinear dynamical systems, including very recent and incomplete attempts by others to assess the possible chaotic behavior observed in laboratory tests.
The term combustion instability has historical roots, and although apparently descriptive, it is misleading. Unstable burning is not the central issue and, in fact, rarely occurs in combustion chambers. Combustion processes provide the source of energy, but what is unstable is the entire system comprising the combusting reagents or propellants and the combustion products forming the medium that supports the unsteady motions. Instabilities arise because the medium and the burning are coupled: not only does the burning supply the medium, but the combustion processes are sensitive to fluctuations of the pressure, temperature, density, and velocity of the environment.

We may view the situation abstractly as analogous to a feedback amplifier in which the addition of feedback to a stable oscillator can produce unstable oscillations. Here the oscillator is the combustion chamber or, more precisely, the unsteady field (pressure velocity, etc.) within the chamber, including various energy losses; feedback is associated with interactions between the unsteady motions and the combustion processes. The situation is schematically shown in Fig. 1. We shall see how the theoretical description of combustion instabilities can be put in a form explicitly having this structure.

The output shown in Fig. 1 is, for example, the pressure observed in the chamber. Although normally there is no input to the system, the possibility is suggested in Fig. 1 because there are important cases in practice when external inputs are applied, usually in the form of pulses generated by igniting small explosive charges. This has long been a standard method for assessing the stability of liquid propellant rockets. ${ }^{1}$ Pulses due to sudden combustion of inadvertant collections of liquid propellants or associated with abrupt changes of throttle settings can occur during actual operation. Similarly, in solid rockets, passage of chunks of material, such as insulation, through the nozzle can produce substantial pulses. Quite extensive work has been done on pulsing solid rockets, ${ }^{2-4}$ but the method has not become a routine part of development programs.

Most problems of combustion instabilities in practice occur without initiation by external inputs of any sort. They arise because the system is intrinsically unstable. During operation, the global parameters characterizing a combustion chamber will generally change, and it is quite possible for the system to pass into and out of conditions when small disturbances are unstable. It is

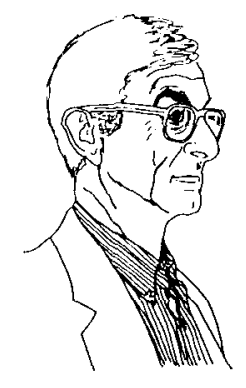

After receiving his Ph.D. from the Massachusetts Institute of Technology in 1961, Professor Culick came to California Institute of Technology where he is currently Professor of Mechanical Engineering and Jet Propulsion. The subject of Culick's dissertation was combustion instabilities in liquid rocket engines; subsequently, he has worked on various problems of unsteady motions in combustion chambers for all types of liquid-fueled and solid-fueled propulsion systems as well as for stationary power plants. His other current research interests include electric propulsion, some aspects of robotics, applied aerodynamics, stability and control, and early aeronautical history. He currently teaches introductory courses on control of mechanical systems and, in collaboration with personnel from Jet Propulsion Laboratory and TRW, on spacecraft design. Professor Culick is a Corresponding Member of the International Academy of Astronautics and a Fellow of the AIAA.

Presented as Paper 90-3927 at the AIAA 13th Aeroacoustics Conference, Tallahassee, FL, Oct. 22-24, 1990; received Jan. 15, 1991; revision received May 10, 1993; accepted for publication May 11, 1993. Copyright $(C) 1990$ by the American Institute of Aeronautics and Astronautics, Inc. All rights reserved. 


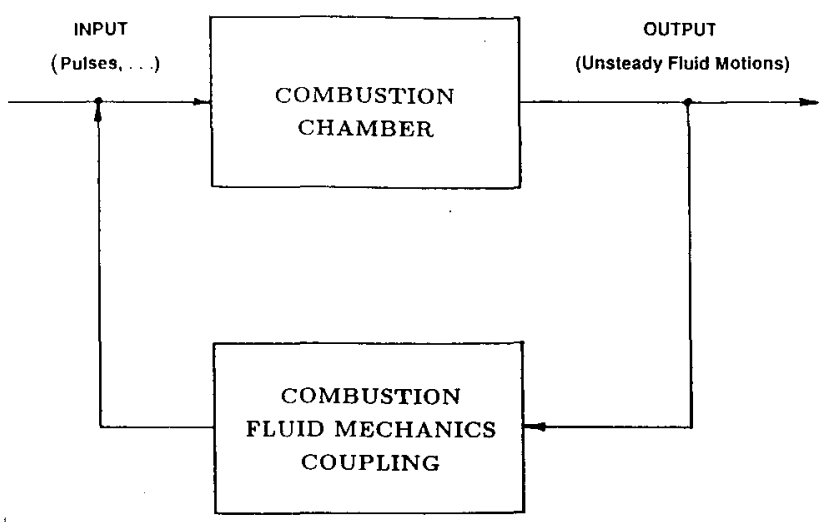

Fig. 1 Schematic representation of combustion instabilities.

that problem, determining the conditions under which the loop in Fig. 1 is unstable, that has received the most attention since studies of combustion instabilities began more than 40 years ago.

The problem of linear instabilities can be treated quite generally if the oscillations are not very different from classical acoustic modes of a chamber having the same shape as that in question but with no flow and rigid walls. ${ }^{5-8}$ The governing equations are linearized, including the influences of the nonuniform flowfield, combustion processes, exhaust nozzle, and other contributions distinguishing this situation from classical acoustics. General formulas can be derived for the frequency shift and growth (or decay) constant, containing all of the information one can obtain from solution to the formal linear problem. Those results are equivalent to finding the roots of the characteristic equation for the feedback system shown in Fig. 1, although a characteristic equation as such does not arise in the formulation normally used to study the stability of combustion chambers.

There are many peripheral theoretical problems that arise, associated with detailed behavior of the combustion processes, boundary conditions, and other characteristics of particular systems. For applications, there are significant difficulties in obtaining necessary quantitative data, for example, for the transfer function representing the combustion/fluid mechanics coupling. Hence, considerable effort is required to obtain quantitative results for the stability of a system, and there are always significant uncertainties. Nevertheless, for the purposes of this paper we may assume that the general behavior of linear combustion instabilities is essentially understood.

Matters are quite different in respect to nonlinear behavior. If the system is linearly unstable, the amplitudes of small disturbances grow exponentially in time, without limit. Nonlinear processes must act if a finite limiting motion is to be reached. Moreover, the behavior of pulses introduced in a chamber, as described earlier, cannot be understood without analyzing nonlinear behavior. Fortunately, the formulation which has become the accepted basis for approximate solution to the linear stability problem can be extended to accommodate nonlinear processes. The main purposes of this paper are to describe that formulation and to summarize some results obtained in the past several years by the author and his colleagues. We shall be concerned primarily with approximate analysis, not numerical solutions to the partial differential equations of conservation. Moreover, as the title suggests, the emphasis is on some problems of acoustics, thereby excluding virtually all of the work devoted to combustion processes and mechanisms that ultimately are responsible for the instabilities. Thus we shall be discussing characteristics of the behavior possible in an unstable system.

The formulation of the equations of motion is described in the next section. Then the basis for the approximate analysis is established with application of the methods of spatial and time averaging to produce ordinary differential equations for the time evolution of the fluctuations. Following a brief summary of previous work on nonlinear combustion instabilities, the remainder of the paper is concerned with several topics illustrating results obtained for special systems and for quite general problems.

\section{Equations of Motion and Formulation of an Approximate Method}

Most combustion chambers contain condensed material, either as liquid propellants or as condensed products of combustion. Particularly in solid rockets there are often considerable amounts of those species: their volume is always a small fraction of that occupied by gases, but the mass fraction is often as large as 0.4 or more. The most important first-order effect of the condensed matter is a reduction of the speed of sound, being determined by the total amount of mass per unit volume (i.e., gas plus liquid and solid material) and the elasticity of the gas. Hence, for analysis of combustion instabilities it is essential to account for condensed species in the conservation equations. Experience has shown that it is sufficient to approximate the medium as one average gas and one average condensed species. Hence, we begin with the full equations of conservation for a two-phase flow and then simplify to the forms suitable to the classes of instabilities we shall subsequently investigate.

\section{A. Equations for Two-Phase Flow}

We allow for the conversion of condensed material to gas at the rate $w_{p}$ (mass/time volume), so the primitive conservation equations are as follows.

Mass (gas):

$$
\frac{\partial \rho_{g}}{\partial t}+\nabla \cdot\left(\rho_{g} u\right)=w_{p}
$$

Mass (particles):

$$
\frac{\partial \rho_{p}}{\partial t}+\nabla \cdot\left(\rho_{p} u_{p}\right)=-w_{p}
$$

Momentum:

$$
\frac{\partial}{\partial t}\left(\rho_{g} u+\rho_{p} u_{p}\right)+\nabla \cdot\left(\rho_{g} u \boldsymbol{u}+\rho_{p} u_{p} u_{p}\right)+\nabla_{p}=\nabla \cdot \hat{\tau}_{v}
$$

$$
\begin{aligned}
& \text { Energy: } \\
& \frac{\partial}{\partial t}\left(\rho_{g} e_{0}+\rho_{p} e_{p 0}\right)+\nabla \cdot\left(\rho_{g} u e_{0}+\rho_{p} u_{p} e_{p 0}\right)+\nabla \cdot(p u)=Q
\end{aligned}
$$

where $\rho_{p}$ stands for the mass of condensed material in unit volume of chamber. The viscous stress tensor for the gases is $\hat{\tau}_{v}$, and $Q$ represents both heat released in chemical reactions and heat transferred by conduction.

The idea now is to combine these equations into forms describing motions in a medium having the mass-averaged properties of the two phases. It is also convenient to combine the mass and energy equations to form an equation for the pressure. After some manipulations and rearrangements (see, e.g., Marble ${ }^{9}$ ), the equations can be put in the form

$$
\begin{gathered}
\frac{\mathrm{D} \rho}{\mathrm{D} t}=\mathscr{W} \\
\rho \frac{\mathrm{D} u}{\mathrm{D} t}=-\nabla p+\mathcal{F} \\
\rho \bar{C}_{v} \frac{\mathrm{D} T}{\mathrm{D} t}=-p \nabla \cdot u+\mathcal{Q} \\
\frac{\mathrm{D} p}{\mathrm{D} t}=-\bar{\gamma} p \nabla \cdot u+\mathscr{P}
\end{gathered}
$$

where

$$
\begin{gathered}
\mathscr{W}=-\rho \nabla \cdot u-\nabla \cdot\left(\rho_{p} \delta u_{p}\right) \\
\mathcal{F}=\nabla \cdot \hat{\tau}_{v}+\delta \mathcal{F}_{p}+\delta u_{p} w_{p}
\end{gathered}
$$




$$
\begin{gathered}
\mathscr{Q}=Q+\delta Q_{p}+\delta u_{p} \cdot \mathcal{F}_{p}+\delta e_{p 0} w_{p}-u \cdot \nabla \cdot \hat{\tau}_{v} \\
\mathscr{P}=\left(\bar{R} / \bar{C}_{\nu}\right)\left[2-\bar{C}_{\nu} T \nabla \cdot\left(\rho_{p} \delta u_{p}\right)\right]
\end{gathered}
$$

The density of the medium is $\rho=\rho_{g}+\rho_{p}$; with $C$ the specific heat of the condensed material (for which $C_{p}=C_{v}=C$ is assumed) and $C_{m}=\rho_{p} / \rho_{g}$, the mass-averaged properties are

$$
\begin{gathered}
\bar{C}_{v}=\frac{C_{v}+C_{m} C}{1+C_{m}} \\
\bar{C}_{p}=\frac{C_{p}+C_{m} C}{1+C_{m}} \\
\bar{\gamma}=\frac{\bar{C}_{p}}{\bar{C}_{v}} \\
\bar{R}=\bar{C}_{p}-\bar{C}_{v}
\end{gathered}
$$

To form Eq. (8) we used the equation of state for the mixture,

$$
p=\rho \bar{R} T
$$

which implies the speed of sound,

$$
a=(\bar{\gamma} \bar{R} T)^{1 / 2}=\left[\frac{\bar{\gamma}}{1+C_{m}}\left(\frac{p}{\rho_{g}}\right)\right]^{1 / 2}
$$

There is some flexibility in representing the coupling between the condensed and gaseous phases. The formulation here rests on the following definitions:

$$
\begin{gathered}
\boldsymbol{F}_{p}=-\rho_{p}\left[\frac{\partial \boldsymbol{u}_{p}}{\partial t}+u_{p} \cdot \nabla u_{p}\right] \\
Q_{p}=-\rho_{p} C\left[\frac{\partial T_{p}}{\partial t}+u_{p} \cdot \nabla T_{p}\right] \\
\delta \boldsymbol{F}_{p}=-\rho_{p}\left[\frac{\partial \delta u_{p}}{\partial t}+\delta u_{p} \cdot \nabla \delta u_{p}+\delta u_{p} \cdot \nabla u+u \cdot \nabla \delta u_{p}\right] \\
\delta Q_{p}=-\rho_{p} C\left[\frac{\partial \delta T_{p}}{\partial t}+\delta u_{p} \cdot \nabla \delta T_{p}+\delta u_{p} \cdot \nabla T+u \cdot \nabla \delta T_{p}\right]
\end{gathered}
$$

and $\delta u_{p}=u_{p}-u, \delta T_{p}=T_{p}-T$. If all of the particles of condensed material have the same size, then $\boldsymbol{u}_{p}$ is the velocity of the typical particle and $T_{p}$ is a characteristic bulk temperature of the typical particle. Equations (16a) and (16b) must be solved for the motion of a typical particle when the local state $(u, T)$ of the medium is specified. Equations (16c) and (16d) give $\delta F_{p}$ and $\delta Q_{p}$ for use in Eqs. (9-12). Although $\delta F_{p}$ and $\delta Q_{p}$ contain nonlinear terms, their influence on combustion instabilities has not been examined thoroughly; limited results suggest that their effects are relatively small and not significant in the sense that they do not affect the qualitative behavior. In practice, the particle sizes have a distribution; a property which is easily taken into account for linear behavior by suitable averaging.

For some purposes, the equation for the entropy is useful. Beginning with the definition $\mathrm{d} s=\left(\mathrm{d} e-p d \rho / \rho^{2}\right) / T$, we can combine the preceding equations to give

$$
\frac{\mathrm{D} s}{\mathrm{D} t}=\mathfrak{s}
$$

where

$$
\mathfrak{S}=\frac{\bar{C}_{v}}{p} \mathscr{P}+\frac{\bar{C}_{p}}{\rho} \nabla \cdot\left(\rho_{p} \delta u_{p}\right)
$$

Within the weak assumptions cited earlier, these equations are quite general. To obtain useful information, it is necessary to simplify them, following the usual practice of writing all of the flow variables as sums of average values and fluctuations and then extracting suitable sets of equations for the fluctuations.

Although we shall not address the subject here, we should note that the given system of equations and the formulation of the approximate analysis discussed next serve well as part of the foundation for a theory of active control of combustion systems. Control can be exerted only by affecting the mass and momentum energy in a flow, processes that may be accommodated by adding source terms $W_{c}, \ldots$, representing the influences of actuator in Eqs. (5-8). The fundamental problem then arises of modeling the actuation process (see Fung et al. ${ }^{10}$ ).

\section{B. Formulation of the Approximate Analysis for Second-Order Acoustics: Spatial and Time Averaging}

Although the set of equations just derived can be used as the basis for numerical analysis of combustion instabilities, no threedimensional problems have been solved numerically. Some progress has been made with one- and two-dimensional problems, summarized briefly later. We are chiefly concerned here with construction of an approximate analysis applicable to general threedimensional problems. In this context, the results serve as an aid to interpreting numerical results. On the other hand, numerical results provide the only means for assessing the accuracy of the approximated analysis, if both methods are applied to the same problems. At the present stage of development, experimental data contain too many uncertainties to be useful in checking the quantitative validity of theoretical results.

Any approximate method begins formally with reduction of the full conservation equations to a set having nonlinearities of lower order. The standard strategy is based on writing all of the variables as sums of mean and fluctuating parts, $p=\bar{p}+p^{\prime}$, etc. To simplify writing, we shall not display the details of the source terms $70, \mathcal{F}, \mathcal{2}$ and $\mathscr{P}$ and to simplify the derivations, we assume that mean values are independent of time. This is not an essential assumption, but correction requires much elaboration. There is a small amount of evidence suggesting that slowly varying mean values, coupled to the unsteady field, may be an important feature of combustion instabilities, but no definite conclusions have been reached. As a reviewer has correctly pointed out, there are many unanswered questions arising in this procedure, including those of closure and of distinguishing acoustical fluctuations from others. Chu and Kovasznay ${ }^{11}$ have given the most complete discussion for linear disturbances, but no thorough treatment exists for nonlinear motions.

Written to second order in the fluctuations, Eqs. (6) and (8) become

$$
\begin{gathered}
\bar{\rho} \frac{\partial u^{\prime}}{\partial t}+\nabla p^{\prime}=-\bar{\rho}\left(\bar{u} \cdot \nabla u^{\prime}+u^{\prime} \cdot \nabla \bar{u}\right) \\
=-\bar{\rho}\left(u^{\prime} \cdot \nabla u^{\prime}\right)-\rho^{\prime} \frac{\partial u^{\prime}}{\partial t}+\mathcal{F} \\
\frac{\partial p^{\prime}}{\partial t}+\bar{\gamma} \bar{p} \nabla \cdot u^{\prime}=-\bar{u} \cdot \nabla p^{\prime}-\bar{\gamma} p^{\prime} \nabla \cdot \bar{u} \\
-u^{\prime} \cdot \nabla p^{\prime}-\bar{\gamma} p^{\prime} \nabla \cdot u^{\prime}+\mathscr{P}^{\prime}
\end{gathered}
$$

Spatial nonuniformities of the mean velocity must be accounted for, but we assume that the average pressure is uniform. That amounts to assuming that the average Mach number is small, so terms of order $\left.|\overline{\boldsymbol{u}}|^{2} \mid \boldsymbol{u}^{\prime}\right\}$ and $\left\{\left.\overline{\boldsymbol{u}}|| \boldsymbol{u}^{\prime}\right|^{2}\right.$ are negligible. Recent analysis described later suggests that nonlinear terms of the second type may in fact be very significant to the qualitative behavior. 
We choose to work with Eqs. (19) and (20) to maintain clear correspondence with classical acoustics. In most of what follows, the familiar classical description can be recovered at any time by setting the mean flow velocity equal to zero. To Eqs. (19) and (20) we must add the equation for the density fluctuation $\rho^{\prime}$, deduced from Eq. (5), and the perturbed form of the equation of state (14). The completed system then consists of six equations for $p^{\prime}, \rho^{\prime}, T^{\prime}$, and the three components of $\boldsymbol{u}^{\prime}$.

Because combustion instabilities are closely related to classical wave motions in a chamber, we form a wave equation for the pressure by differentiating Eq. (20) with respect to time and substituting Eq. (19) for $\partial u^{\prime} / \partial t$ to give the result

$$
\nabla^{2} p^{\prime}-\frac{1}{\bar{a}^{2}} \frac{\partial^{2} p^{\prime}}{\partial t^{2}}=h
$$

where $\bar{a}^{2}=\bar{\gamma} \bar{R} \bar{T}$ is assumed independent of position and

$$
\begin{aligned}
h= & -\bar{\rho} \nabla \cdot\left(\bar{u} \cdot \nabla u^{\prime}+u^{\prime} \cdot \nabla \bar{u}\right)+\frac{1}{\bar{a}^{2}} \bar{u} \cdot \nabla \frac{\partial p^{\prime}}{\partial t} \\
& +\frac{\bar{\gamma}}{\bar{a}^{2}} \frac{\partial p^{\prime}}{\partial t} \nabla \cdot \bar{u}-\nabla \cdot\left(\bar{\rho} u^{\prime} \cdot \nabla u^{\prime}+\rho^{\prime} \frac{\partial u^{\prime}}{\partial t}\right) \\
& +\frac{1}{\bar{a}^{2}} \frac{\partial}{\partial t}\left(u^{\prime} \cdot \nabla p^{\prime}\right)+\frac{\bar{\gamma}}{\bar{a}^{2}} \frac{\partial}{\partial t}\left(p^{\prime} \nabla \cdot u^{\prime}\right)+\nabla \cdot \mathfrak{F}-\frac{1}{\bar{a}^{2}} \frac{\partial \mathscr{P}^{\prime}}{\partial t}
\end{aligned}
$$

The boundary condition is set on the gradient of $p^{\prime}$, found by taking the scalar product of the outward normal vector with Eq. (20)

$$
\hat{n} \cdot \nabla p^{\prime}=-f
$$

and

$$
\begin{aligned}
f= & \bar{\rho} \frac{\partial u^{\prime}}{\partial t} \cdot \hat{n}+\bar{\rho}\left(\bar{u} \cdot \nabla u^{\prime}+u^{\prime} \cdot \nabla \bar{u}\right) \cdot \hat{n} \\
& +\bar{\rho}\left(u^{\prime} \cdot \nabla u^{\prime}\right) \cdot \hat{n}+\rho^{\prime} \frac{\partial u^{\prime}}{\partial t} \cdot \hat{n}-\mathcal{F}^{\prime} \cdot \hat{n}
\end{aligned}
$$

The basis of the approximate method is replacement of the partial differential equations of conservation by an equivalent system of ordinary differential equations. This is accomplished by averaging over the volume of the chamber, using essentially Galerkin's method. First multiply the equations by a suitable weighting function, taken to be a mode shape for unperturbed motions, and then integrate over the volume. The unperturbed waves are governed by Eqs. (21) and (23) with $h=f=0$. If the Mach number in the chamber is high and the influence of the exhaust nozzle is large, this may be a poor choice for the weighting function.

Appropriate corrections can be made, at the expense of much increased labor. Here we base the analysis on the limiting case of no perturbations; then the mode shape $\psi_{n}$ for the mil mode satisfies the equations

$$
\begin{gathered}
\nabla^{2} \psi_{n}+k_{n}^{2} \Psi_{n}=0 \\
\hat{n} \cdot \nabla \Psi_{n}=0
\end{gathered}
$$

We seek a description which amounts to comparing the unperturbed problem with the actual problem to be analyzed. Multiply Eq. (21) by $\Psi_{n}$, multiply Eq. (25) by $p^{\prime}$, subtract the results, and integrate over the chamber

$$
\begin{aligned}
& \int\left[\Psi_{n} \nabla^{2} p^{\prime}-p^{\prime} \nabla^{2} \Psi_{n}\right] \mathrm{d} V-\frac{1}{a^{2}} \int \Psi_{n} \frac{\partial^{2} p^{\prime}}{\partial t^{2}} \mathrm{~d} V \\
& -k_{n}^{2} \int p^{\prime} \nabla^{2} \Psi_{n} \mathrm{~d} V=\int \Psi_{n} h \mathrm{~d} V
\end{aligned}
$$

Apply Green's theorem to the left-hand side, substitute the boundary conditions (23) and (25b), and rearrange the terms to give

$$
-\frac{1}{\bar{a}^{2}} \int \psi_{n} \frac{\partial^{2} p^{\prime}}{\partial t^{2}} \mathrm{~d} V-k_{n}^{2} \int \psi_{n} p^{\prime} \mathrm{d} V=\int \Psi_{n} h \mathrm{~d} V+\iint \Psi_{n} f \mathrm{~d} V(26)
$$

We assume that the normal modes $\psi_{m}(r)$ for a complete orthogonal set, suitable as a basis for expressing the unsteady pressure field as a series with time varying amplitudes $\eta_{m}(t)$

$$
p^{\prime}(r, t)=\bar{p} \sum_{m=1}^{\infty} \eta_{m}(t) \Psi_{m}(\boldsymbol{r})
$$

For classical acoustics, the velocity in a normal mode is $\dot{n}_{m}(t) \nabla \psi_{m}(r) / \bar{\gamma} k_{m}^{2}$; hence, corresponding to Eq. (27) we write the velocity field as

$$
\boldsymbol{u}^{\prime}(\boldsymbol{r}, t)=\sum_{m=1}^{\infty} \frac{\dot{\eta}_{m}(t)}{\bar{\gamma} k_{m}^{2}} \nabla \Psi_{m}(\boldsymbol{r})
$$

These are clearly not exact representations of the true fields, for the boundary conditions are not satisfied. Equation (27) gives $\hat{n} \cdot \nabla p^{\prime}=0$ because $\hat{n} \cdot \nabla \psi_{m}=0$, and according to Eq. (28) the velocity fluctuation vanishes on the boundary. The spatial structure of the unsteady motions near the boundary is not accurately reproduced. Nevertheless, the errors are small when the perturbations represented by $h$ and $f$ are small. That means, in particular, that any admittance function introduced to represent behavior of a boundary must be small. Because of the spatial averaging, the equations to be found for the amplitudes will provide a satisfactory basis for studying real problems, a claim justified partly by comparison of approximate results with numerical solution to the conservation equations for a limited number of one-dimensional problems.

In some sense, the representation Eq. (28) of the velocity is restricted a priori because the same functions $\eta_{m}(t)$ appear there as in Eq. (27) for the pressure: some flexibility has evidently been lost. The form (28) has been used principally because, with Eq. (27), the correct results are necessarily obtained in the limit of classical acoustics, $h, f \rightarrow \infty$. We can expect, in any case, that the analysis worked out here should be valid only when the perturbations are small, but presently we have no direct means of assessing the errors due to the use of Eq. (28).

Because we assume that the normal modes are orthogonal, they have the property

$$
\begin{gathered}
\int \Psi_{m} \Psi_{n} \mathrm{~d} V=E_{n}^{2} \delta_{m n} \\
E_{n}^{2}=\int \Psi_{n}^{2} \mathrm{~d} V
\end{gathered}
$$

Now substitute Eq. (27) in the left-hand side of Eq. (26) and use Eqs. (29a) and (29b) to find the system of equations for the amplitudes

$$
\frac{\mathrm{d}^{2} \eta_{n}}{\mathrm{~d} t^{2}}+\omega_{n}^{2} \eta_{n}=F_{n}
$$

where $\omega_{n}=\bar{a} k_{n}$ and

$$
F_{n}=-\frac{a^{-2}}{\bar{p} E_{n}^{2}}\left\{\int h \psi_{n} \mathrm{~d} V+\iint f \psi_{n} \mathrm{~d} S\right\}
$$

The functions $h$ and $f$ contain $p^{\prime}$ and $\boldsymbol{u}^{\prime}$, to be replaced by the expansions (27) and (28). It is at that point that any errors due to the assumed form (28) will enter. It is a simple matter to introduce 
a different representation of the acoustic velocity, but some of the later results will become more complicated when the right-hand side of Eq. (31) is evaluated.

The "force" $F_{n}$ will in any case depend on the amplitudes $\eta_{m}(t)$. In general, both linear and nonlinear terms will appear, and Eq. (30) describes the time evolution of a collection of coupled nonlinear oscillators, one oscillator being associated with each classical mode.

Analysis is already simplified by the progression from partial to ordinary differential equations. We could apply the system (30) directly, as Zinn and Powell ${ }^{12,13}$ first did in a similar treatment of combustion instabilities in liquid rockets. However, we choose to apply the method of time averaging and replace the system of second-order equations by a corresponding system of first-order equations. Not only is the cost of routine calculations reduced, but this step provides a convenient basis for formally studying the general behavior of unsteady motions.

Time averaging is a reasonable procedure for the classes of problems we are most concerned with. The oscillations observed in practice commonly have amplitudes and phases slowly varying in time: their fractional changes are small during one period of oscillation. Hence, the amplitudes $\eta_{n}(t)$ may be written in the form

$$
\eta_{n}(t)=r_{n}(t) \sin \left[\omega_{n} t+\phi_{n}(t)\right]=A_{n}(t) \sin \omega_{n} t+B_{n}(t) \cos \omega_{n} t
$$

The time-varying phase $\phi_{n}(t)$ is observed as a frequency shift. the actual frequency for the perturbed mode being $\omega_{n}+\dot{\phi}_{n}$. Construction of the equations for $r_{n}(t)$ and $\phi_{n}(t)$, or equivalently $A_{n}(t)$ and $B_{n}(t)$, may be done in several ways; we shall simply quote the results here.

Substitution of the assumed form (32) for $\eta_{n}$ in Eq. (30), and imposition of the constraint

$$
\frac{\mathrm{d} A_{n}}{\mathrm{~d} t} \sin \omega_{n} t+\frac{\mathrm{d} B_{n}}{\mathrm{~d} t} \cos \omega_{n} t=0
$$

leads to the two equations

$$
\begin{aligned}
& \frac{\mathrm{d} A_{n}}{\mathrm{~d} t}=\frac{1}{\omega_{n}} F_{n} \cos \omega_{n} t \\
& \frac{\mathrm{d} B_{n}}{\mathrm{~d} t}=-\frac{1}{\omega_{n}} F_{n} \sin \omega_{n} t
\end{aligned}
$$

These equations are entirely equivalent to $\mathrm{Eq}$. (30). We have the freedom to satisfy Eq. (33) because two functions $A_{n}(t)$ and $B_{n}(t)$ have been introduced in place of $\eta_{n}(t)$. Now average Eqs. (34a) and (34b) over an interval $\tau$ including many periods of the oscillation $\left(\tau » 2 \pi / \omega_{n}\right)$, but during which $A_{n}(t)$ and $B_{n}(t)$ are nearly constant, giving

$$
\begin{aligned}
& \frac{\mathrm{d} A_{n}}{\mathrm{~d} t}=\frac{1}{\omega_{n}} \int_{t}^{(t+\tau)} F_{n} \cos \omega_{n} t^{\prime} \mathrm{d} t^{\prime} \\
& \frac{\mathrm{d} B_{n}}{\mathrm{~d} t}=-\frac{1}{\omega_{n}} \int_{t}^{(t+\tau)} F_{n} \sin \omega_{n} t^{\prime} \mathrm{d} t^{\prime}
\end{aligned}
$$

More precisely, we should explicitly recognize the presence of two time scales: a fast scale $\tau_{n}=2 \pi / \omega_{n}$ and a slow scale characterizing the changes of $A_{n}$ and $B_{n}$. Then two dimensionless time variables can be defined, the fast time $t_{f}=\omega_{n} t$ and the slow time $t_{s}=\varepsilon t_{f}$ where $\varepsilon$ is a measure of the smallness of the perturbing force $F_{n}$.
The force is a function of both the fast and slow variables because it depends on the $\eta_{i}$ and

$$
\eta_{i}=A_{i}\left(t_{s}\right) \sin \left(\frac{\omega_{i}}{\omega_{n}} t_{f}\right)+B_{i}\left(t_{s}\right) \cos \left(\frac{\omega_{i}}{\omega_{n}} t_{f}\right)
$$

When the right-hand sides of Eqs. (35a) and (35b) are expressed in terms of these two variables, the equations are

$$
\begin{aligned}
& \frac{\mathrm{d} A_{n}}{\mathrm{~d} t}=\frac{1}{\omega_{n}} \int_{t_{f}}^{t_{f}^{+} \tau_{f}} F_{n}\left(t_{s}^{\prime}, t_{f}^{\prime}\right) \cos t_{f}^{\prime} \mathrm{d} t_{f}^{\prime} \\
& \frac{\mathrm{d} B_{n}}{\mathrm{~d} t}=\frac{-1}{\omega_{n}} \int_{t_{f}}^{t_{f}^{+\tau_{f}}} F_{n}\left(t_{s}^{\prime}, t_{f}^{\prime}\right) \sin t_{f}^{\prime} \mathrm{d} t_{f}^{\prime}
\end{aligned}
$$

For second-order acoustics, $F_{n}$ has the form ${ }^{5}$

$$
F_{n}=-\sum_{i=1}^{\infty}\left[D_{n i} \dot{\eta}_{i}+E_{n i} \eta_{i}\right]-\sum_{i=1}^{\infty} \sum_{j=1}^{\infty}\left[A_{n_{i j}} \dot{\eta}_{i} \dot{\eta}_{j}+B_{n_{i j}} \eta_{i} \eta_{j}\right]
$$

The coefficients $D_{n_{i}}, \ldots$, depend on the unperturbed mode shapes and frequencies; the $D_{n}$ and $E_{n}$ arise from linear processes and are usually proportional to the average Mach number. (A notable exception are the contributions from condensed material.) When Eq. (37) is substituted in Eqs. (36a) and (36b), the averaging must be carried out over terms oscillating at frequencies which are sums and differences of the natural frequencies. Except in special cases the averaging process does not automatically dictate which tern:s should be dropped because their averaged values are negligibly small. Some experience with particular problems ${ }^{14}$ suggests that if the frequency of a term is less than half of the frequency of the mode in question, then that term should be retained. We shall see an example in Sec. VI dealing with transverse modes.

This method of time averaging necessarily brings with it errors relative to the results obtained directly with the second-order equations (30). We shall discuss the matter briefly in Sec. IX.

\section{Brief Review of Previous Analyses}

The formulation described in the preceding section has its roots in two papers published in the $1970 \mathrm{~s}^{5,15}$ Long before those works, much attention had been directed to nonlinear combustion instabilities. That the phenomena are intrinsically nonlinear was recognized practically from the earliest experimental results. In 1956 , motivated partly by the common occurrence of transverse waves in combustors, Maslen and Moore ${ }^{16}$ treated finite amplitude waves, based on a power series expansion in the amplitude, but with no combustion and mean flow. Flandro ${ }^{17}$ extended that work to include combustion and flow, and succeeded in explaining the origin of roli torques observed occasionally in solid rockets.

$\mathrm{Chu}^{18}$ and $\mathrm{Chu}$ and $\mathrm{Ying}^{19}$ treated the problem of thermally driven nonlinear longitudinal oscillations with shock waves in a closed tube using the method of characteristics. Although the heat source was allowed to be sensitive to pressure in part of that work, no combustion and flow were accounted for.

Motivated partly by laboratory tests of gas-fueled rockets at Princeton, the first detailed analysis of the problem with combustion and flow was published by Sirignano ${ }^{20}$ and Sirignano and Crocco, ${ }^{21}$ also using the method of characteristics, modified to include coordinate stretching following the Poincare-LighthillKuo (PLK) procedure. That work was followed by further analyses of longitudinal waves by Mitchell et $\mathrm{al} .^{22}$ and by Crocco and Mitchell ${ }^{23}$ using different methods allowing treatment of distributed combustion. At the same time, Zinn ${ }^{24}$ examined nonlinear behavior of transverse modes, based on expansion of the equation for the velocity potential in powers of the amplitude. 
Those early works by the Princeton group were first to address quantitatively the broad subject of nonlinear motions in a combustion chamber. They were also first to expose some particular problems which remain unsolved in general, notably the possible existence of stable limit cycles and the conditions under which "triggering" to stable or unstable limit cycles may occur. However, apparently for several reasons, the approaches taken during the 1960 s have not been pursued further by researchers outside the Princeton school. Perhaps foremost, the calculations become quite involved, requiring restrictive approximations to obtain results. For example, in some of the analyses, ${ }^{22-24}$ the time lag or $n-\tau$ model of the combustion dynamics was used, whereas others ${ }^{20,21}$ incorporated a kinetics model of the chemical dynamics, with no time lag and, hence, no dependence on frequency. The approximation of a thin combustion zone was made in some $\operatorname{cases}^{20-22,24}$ but a special form of distributed combustion was treated by Crocco and Mitchell. ${ }^{23}$ It is the nature of the analytical methods that such well-defined approximations must be made, but it is subsequently difficult to assess the extent to which the predicted nonlinear behavior depends on those approximations. No attempt to sort out the differences has been published. The involved character of the analyses and the difficulties of understanding the physical behavior for application in practical routine fashion seems to have discouraged wider use of the methods.

Subsequent to the works just cited, Hardesty, ${ }^{25}$ also at Princeton, carried out a lengthy series of tests with a laboratory rocket fueled by premixed gases. An interesting aspect of that system is that under some conditions, time lags associated with finite kinetics are important. It might be useful, in view of theoretical work accomplished more recently, to re-examine those experimental results, but that matter lies outside the intentions of this review.

Nonlinear behavior of a dynamical system is both qualitatively and quantitatively dependent on the values of the parameters characterizing the linear behavior. Generalizations from particular results are almost never possible without actually performing the necessary analysis. Therefore, there is presently almost no basis for claiming generality for any of the results involving the time lag or infinitely fast kinetic models of linear behavior, however useful the model may be for other purposes. Similarly, much more work would have to be done to clarify the sensitivity of the results cited to the specialized distributions of combustion.

The introduction by Zinn and Powell ${ }^{13}$ of the use of Galerkin's method to study instabilities in liquid rockets, and later applied by Zinn and Lores ${ }^{26}$ and Lores and Zinn, ${ }^{27}$ marked an important shift of emphasis on the type of analytical methods used in this field. As in the earlier works, the time-lag model of combustion is used, so the results are similarly restricted. However, the general approach, pursued independently by Culick ${ }^{5,15}$ for application to solid rockets, will accommodate very general representations of all of the nonlinear processes. One important purpose of the analyses reported in the remainder of this paper has been to clarify those aspects of nonlinear behavior that can be attributed to nonlinear gasdynamics, the most accurately represented process in the problems treated here. The quantities $D_{n_{i}}, E_{n_{i}}$ arising from linear processes in Eq. (37) are assigned values but are not computed for any particular processes.

\section{Linear Stability}

Stability of small disturbances is the most important linear problem in the study of combustion instabilities. Steady waves in combustion chambers necessarily involve nonlinear processes. Accordingly, a great deal of effort has been devoted to studying the transient behavior of disturbances, particularly in solid rockets. Much data has been collected, allowing satisfactory confirmation of the essential ideas, including the relative magnitude of the various contributions to energy gains and losses. The standard method for computing the stability of solid rockets is based on the approximate analysis described here; it has been developed into a widely used computer program originally prepared by Lovine et al. ${ }^{28}$ and recently revised by Nickerson et al. ${ }^{29}$ Before we discuss nonlinear problems, it is useful to show how the limiting case of linear stability is treated.
There really is only one stability problem, namely, computation of the complex wave number, $k=(\omega-i \alpha) / \bar{a}$ for a steady wave. The real part gives the actual frequency, shifted from the frequency of a classical mode by the linear perturbations, and the imaginary part gives the growth or decay constant $\alpha$. If $\alpha$ is positive, the mode in question is unstable.

For these calculations, only the linear terms in $h$, Eq. (22), and $f$, Eq. (24), are retained. Hence, the forcing function $F_{n}$ defined in Eqs. (30) and (31) is linear in $p^{\prime}$ and $\boldsymbol{u}^{\prime}$; after time averaging, $F_{n}$ has the form of Eq. (37), but all of the $A_{n_{i j}}$ and $B_{n_{i j}}$ are zero. As usual for computing stability, we assume exponential time dependence and write

$$
\eta_{n}=\hat{\eta}_{n} e^{i \bar{a} k t} \quad h=\hat{h}^{i \bar{a} k t} \quad f=\hat{f} e^{i \bar{a} k t}
$$

In general, one must expect that the off-diagonal coefficients $D_{n_{i}}$ and $E_{n_{i}}$ are nonzero. Substitution in Eq. (30) with $F_{n}=-\Sigma$ $\left(D_{n_{i}} \dot{\eta}_{i}+E_{n_{i}} \eta_{i}\right)$ and cancellation of the common factor $e^{i a k t}$ then leads, in the usual way, to a characteristic polynomial of order $N$ in $\bar{a} k^{2}$, where $N$ is the number of nodes considered. The corresponding orthogonal modes and frequencies should then be used to reform the Galerkin expansion (27) for use in nonlinear problems. However, it appears that, usually in problems of combustion instabilities, linear coupling between modes is either absent or so small as to have no measurable influences. Therefore, we set $D_{n_{i}}=E_{n_{i}}$ $=0$ and Eq. (30) becomes

$$
F_{n}=-D_{n n} \dot{\eta}_{n}-E_{n n} \eta_{n}
$$

Substitution of Eq. (38) in Eq. (30) and cancellation of the common factor $e^{i \bar{a} k t}$ gives the formula for $k^{2}$,

$$
k^{2} \equiv \frac{1}{-a^{2}}(\omega-i \alpha)^{2}=\frac{\omega_{n}^{2}}{\bar{a}^{2}}+\frac{1}{p E_{n}^{2}}\left\{\int \frac{\hat{h}}{\hat{\eta}_{n}} \Psi_{n} \mathrm{~d} V+\iint \frac{\hat{f}}{\hat{\eta}_{n}} \Psi_{n} \mathrm{~d} S\right\}
$$

Taking the real and imaginary parts, and using the fact that both are proportional to the average Mach number, so $\alpha / \omega,\left(\omega-\omega_{n}\right) /$ $\omega_{n} \ll 1$, we find formulas for the actual frequency and the growth constant

$$
\begin{gathered}
\omega=\omega_{n}+\frac{-a^{2}}{2 \omega_{n}^{-} p E_{n}^{2}}\left\{\int \frac{\hat{h}^{(r)}}{\hat{\eta}_{n}} \Psi_{n} \mathrm{~d} V+\iint \frac{\hat{f}^{(r)}}{\hat{\eta}_{n}} \mathrm{~d} S\right\} \\
\alpha=\frac{-\bar{a}^{2}}{2 \omega_{n} \bar{p} E_{n}^{2}}\left\{\int \frac{\hat{h}^{(i)}}{\hat{\eta}_{n}} \Psi_{n} \mathrm{~d} V+\iint \frac{\hat{f}^{(i)}}{\hat{\eta}_{n}} \Psi_{n} \mathrm{~d} S\right\}
\end{gathered}
$$

where superscripts $(r)$ and $(i)$ denote real and imaginary parts, respectively. Thus, the entire problem of linear stability comes down to evaluating the integrals over $\hat{h}$ and $\hat{f}$. This is a perfectly general result restricted only by the assumptions that the $\psi_{n}$ satisfy Eqs. (25a) and (25b) and Eqs. (29a) and (29b), and that linear coupling of the modes is absent. For applications, modeling of the source terms in $h$ and $f$ will eventually bring further restrictions.

With $F_{n}$ given by Eq. (39), if the interval of averaging is taken to be the period of the $n$th mode, the time-averaged forms of Eqs. (36a) and (36b) are

$$
\begin{aligned}
& \left(\frac{\mathrm{d} A_{n}}{\mathrm{~d} t}\right)_{\text {linear }}=-\frac{1}{2} D_{n n} A_{n}-\frac{1}{2} \frac{E_{n n}}{\omega_{n}} B_{n} \\
& \left(\frac{\mathrm{d} B_{n}}{\mathrm{~d} t}\right)_{\text {linear }}=-\frac{1}{2} D_{n n} B_{n}+\frac{1}{2} \frac{E_{n n}}{\omega_{n}} A_{n}
\end{aligned}
$$


Multiply the first of these by $A_{n}$, the second by $B_{n}$, and add the results to find the equation for the amplitude $r_{n}=\left(A_{n}^{2}+B_{n}^{2}\right)^{1 / 2}$

$$
\frac{\mathrm{d} r_{n}}{\mathrm{~d} t}=-\frac{1}{2} D_{n n} r
$$

Thus, $r_{n} \sim e^{\alpha_{n} t}$, with $\alpha_{n}=-D_{n n} / 2$ the growth constant for the $n$th mode. Comparison with Eq. (41b) gives the recipe for calculating $D_{n n}$.

The definition (32) of $\eta_{n}$ gives

$$
A_{n}=e^{\alpha_{n} t} \cos \phi_{n} \quad B_{n}=e^{\alpha_{n t}} \sin \phi_{n}
$$

Substitution in Eqs. (42a) and (42b) leads eventually to the equation for $\phi_{n}$,

$$
\frac{\mathrm{d} \phi_{n}}{\mathrm{~d} t}=\frac{E_{n n}}{2 \omega_{n}}
$$

We have already remarked after Eq. (32) that $\dot{\phi}_{n}$ is the frequency shift, $\omega-\omega_{n}=-\theta_{n}$. Comparison with Eq. (41a) then gives the formula for calculating the coefficient $E_{n n}=2 \omega_{n}\left(\omega-\omega_{n}\right)=-2 \omega_{n} \theta_{n}$.

Most of the effort related to problems of linear stability has been spent on experimental work to obtain information necessary for evaluating the various contributions to the integrals in Eqs. (41a) and $(4 \mathrm{lb})$.

\section{Nonlinear Longitudinal Modes; Limit Cycles}

Purely longitudinal modes have the important special characteristic that the unperturbed natural frequencies are integral multiples of the fundamental. As a result, the double series in $F_{n}$, Eq. (37) reduces to a single series, and the first-order equations obtained with time averaging become

$$
\begin{aligned}
& \frac{\mathrm{d} A_{n}}{\mathrm{~d} t}=\alpha_{n} A_{n}+\theta_{n} B_{n}+\frac{n \beta}{2} \sum_{i=1}^{\infty} \\
& \quad \times\left[A_{i}\left(A_{n-i}-A_{i-n}-A_{i+n}\right)-B_{i}\left(B_{n-i}+B_{i-n}-B_{i+n}\right)\right] \\
& \frac{\mathrm{d} B_{n}}{\mathrm{~d} t}=-\theta_{n} A_{n}+\alpha_{n} B_{n}+\frac{n \beta}{2} \sum_{i=1}^{\infty} \\
& \quad \times\left[A_{i}\left(B_{n-i}+B_{i-n}-B_{i+n}\right)+B_{i}\left(A_{n-i}-A_{i-n}-A_{i+n}\right)\right]
\end{aligned}
$$

where $\alpha_{n}=-D_{n n} / 2, \theta_{n}=-E_{n n} / 2 \omega_{n}$, and

$$
\beta=[(\bar{\gamma}+1) / 8 \bar{\gamma}] \omega_{1}
$$

Although actual systems rarely show $\omega_{n}=n \omega_{1}$ exactly, there are many instances, particularly of solid and liquid rockets, when the spectrum of frequencies closely satisfies this condition. Hence, this special case has received much attention since it was first studied by Culick. ${ }^{5}$ Prior to that time, no formal theory existed to explain the observed occurrences of steady nonlinear periodic oscillations, i.e., limit cycles; calculations of limit cycles had been carried out in earlier works cited in Sec. III, but analytical results had not been obtained owing to the nature of the methods used.

Therefore, most attention was directed to discovering first whether limit cycles could be predicted, and second the conditions determining their existence and stability. Note that the constant $\beta$ can be absorbed as a scaling parameter $\left(\beta^{-1}\right.$ is a time scale) so that all of the behavior-linear and nonlinear-is determined by the values of the linear parameters $\left(\alpha_{n}, \theta_{n}\right)$, which contain completely the influences of all of the linear processes. In the earliest work, the existence of limit cycles was established by solving Eqs. (45a) and (45b) numerically for ranges of the linear parameters. ${ }^{5}$

Confidence in the approximate analysis was gained quite early with successful comparisons and numerical solutions to the partial differential equations. ${ }^{30,31}$ Several problems were examined, including development of a disturbance into a weak shock wave (no combustion or mean flow), decay of large amplitude waves due to the presence of condensed material, and the growth of a combustion instability to a limit cycle in a solid rocket. Using more efficient numerical routines developed by Levine and Baum, ${ }^{32}$ Culick and Yang $^{8}$ have reported the example shown in Fig. 2 showing the waveform in a limit cycle computed with the numerical and approximate analysis (for five modes). The frequencies (shifted due to perturbations) and the amplitudes of the modes are predicted quite well by the approximate analysis as Table 1 shows.

The frequencies agree within $3 \%$, illustrating the familiar fact that accurate prediction of frequencies is not a demanding test of an approximate analysis; good approximations should (as here) be built into the formulation. More to the point is the good agreement of the amplitudes except for the highest $(n=5)$ mode. The reason for the higher approximate values is that truncation to five modes eliminates energy transfer to higher modes where it is ultimately dissipated. We shall see in the following sections that the intrinsic tendency for energy transfer upward is an important fundamental property of these problems, having a strong influence on the development of limit cycles from small initial disturbances.

\section{A. Limit Cycles for Two Modes}

Although it is useful to be able to obtain results of this sortinexpensive and accurate approximations to nonlinear behaviorthis is only part of the story. It is perhaps even more significant, both theoretically and practically, to establish the qualitative dependence of nonlinear behavior on the linear parameters. The special structure of the nonlinear Eqs. (45a) and (45b) allows considerable progress in that respect. There are really two general problems to solve: 1) the conditions for existence and stability of limit cycles, and 2) the conditions under which a linear stable system becomes unstable to a sufficiently large disturbance. The second problem concerns nonlinear instability, a phenomenon that has been given the name triggering, which we shall consider further in Secs. VII and VIII. Awad and Culick ${ }^{33}$ reported the first formal results for existence and stability of limit cycles for purely longitudinal modes, using Eqs. (45a) and (45b), although as noted in Sec. III, the question had been primarily addressed with numerical solutions. Closed-form results have been obtained only for the case of two modes; here we only describe the gist of the matter.

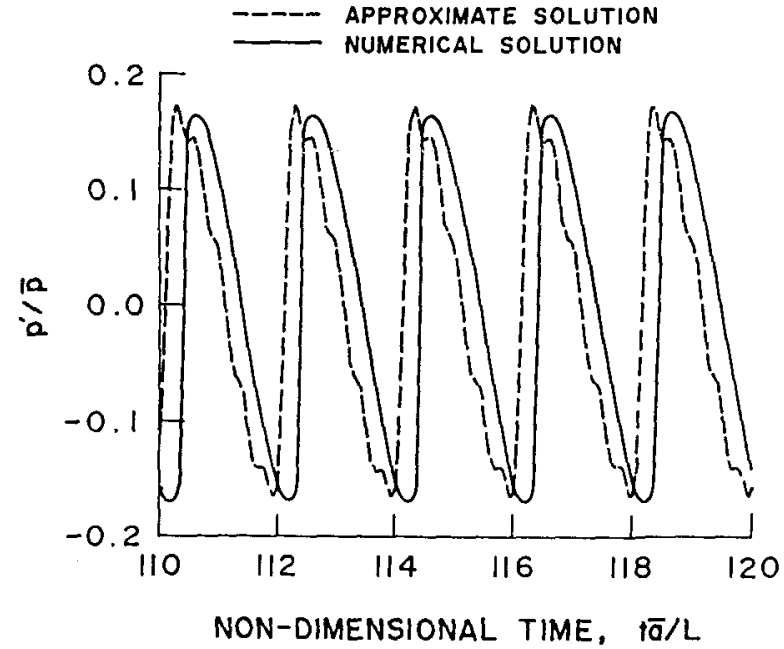

Fig. 2 Comparison of waveforms: numerical and approximate solutions. 
Table 1 Comparison of results for numerical and approximate solutions

\begin{tabular}{lccccc}
\hline & \multicolumn{5}{c}{ Frequency $\left|p^{\prime} / \tilde{p}\right|$} \\
\cline { 2 - 6 } Mode & 1 & 2 & 3 & 4 & 5 \\
Numerical & 926 & 1824 & 2698 & 3595 & 4491 \\
Approximate & 895 & 1785 & 2683 & 3571 & 4449 \\
& \multicolumn{5}{c}{ Amplitude $\left|p^{\prime} / \bar{p}\right|$} \\
Mode & 1 & 2 & 3 & 4 & 5 \\
Numerical & 0.151 & 0.042 & 0.234 & 0.0203 & $\overline{0}$ \\
Approximate & 0.151 & 0.0478 & 0.0280 & 0.0153 & 0.0188 \\
\hline \hline
\end{tabular}

For two modes, Eqs. (45a) and (45b) become

$$
\begin{gathered}
\frac{\mathrm{d} A_{1}}{\mathrm{~d} t}=\alpha_{1} A_{1}+\theta_{1} B_{1}-\beta\left(A_{1} A_{2}-B_{1} B_{2}\right) \\
\frac{\mathrm{d} B_{1}}{\mathrm{~d} t}=\alpha_{1} B_{1}-\theta_{1} A_{1}+\beta\left(B_{1} A_{2}-A_{1} B_{2}\right) \\
\frac{\mathrm{d} A_{2}}{\mathrm{~d} t}=\alpha_{2} A_{2}+\theta_{2} B_{2}+\beta\left(A_{1}^{2}-\beta_{1}^{2}\right) \\
\frac{\mathrm{d} B_{2}}{\mathrm{~d} t}=\alpha_{2} B_{2}-\theta_{2} A_{2}+2 \beta B_{1} A_{1}
\end{gathered}
$$

Limit cycles correspond to equilibrium states for this system. There are two possible cases: $\dot{A}_{n}=\dot{B}_{n}=0$, or $A_{n}=a_{n} \cos \left(v_{n} t+\psi_{n}\right)$, $B_{n}=a_{n} \sin \left(v_{n} t+\psi_{n}\right)$. In the first case, the functions $A_{n}, B_{n}$ are constant in the limit cycle, and in the second case they oscillate with frequencies $v_{n}$. The two possibilities were first discovered in the numerical calculations reported by Culick $^{5}$ and confirmed theoretically by Awad and Culick. ${ }^{33}$

The analysis then proceeds as follows. First substitute in Eqs. (47a-47d) the assumed forms for the $A_{n}, B_{n}$ in the limit cycle and solve the algebraic equations. It is a consequence of the special structure of those equations that formulas for the amplitudes can be derived, simultaneously with conditions for their existence. Let subscript ()$_{0}$ denote values in the limit cycle; then for the case when the amplitudes are constant in the limit cycle, the results may be written

$$
\begin{gathered}
A_{10}=\frac{1}{\beta}\left[-\alpha_{1} \alpha_{2}\left(1+\frac{\theta_{1}^{2}}{\alpha_{1}^{2}}\right)\right]^{1 / 2} \\
B_{10}=0 \\
A_{20}=(1 / \beta) \alpha_{1} \\
B_{20}=(1 / \beta) \theta_{2}
\end{gathered}
$$

These formulas are not unique. Because there is an arbitrary phase on the limit cycle, one constant is undetermined; here its value has been fixed by setting $B_{10}=0$.

If the amplitudes are allowed to oscillate in the limit cycle, producing a small shift in the modal frequencies, then

$$
A_{n}=a_{n} \cos \left(v_{n} t+\psi_{n}\right) ; \quad B_{n}=a_{n} \sin \left(v_{n} t+\psi_{n}\right)
$$

The frequency shifts are found to be

$$
v_{1}+\frac{1}{2} v_{2}=-\frac{\alpha_{1} \theta_{2}+\alpha_{2} \theta_{1}}{2 \alpha_{2}-\alpha_{2}}
$$

and the maximum amplitudes are

$$
\begin{gathered}
\alpha_{1}=\left\{-\frac{\alpha_{1} \alpha_{2}}{\beta_{2}}\left[1+\left(\frac{2 \theta_{1}-\theta_{2}}{2 \alpha_{1}+\alpha_{2}}\right)^{2}\right]\right\}^{1 / 2} \\
\alpha_{2}=\frac{\alpha_{1}}{\beta}\left[1+\left(\frac{2 \theta_{1}-\theta_{2}}{2 \alpha_{1}+\alpha_{2}}\right)^{2}\right]^{1 / 2}
\end{gathered}
$$

Because $A_{10}$ [Eq. (48a)] and $a_{1}$ [Eq. (50a)] must be real, one necessary condition for existence is

$$
\alpha_{1} \alpha_{2}<0
$$

The physical interpretation of this condition follows from the meaning of the $\alpha_{n}$ as growth or decay constants. If $\alpha_{n}$ is positive (negative) then in the linear approximation, energy is added (lost) to the $n$th mode. Hence, Eq. (51) represents the requirement that one mode must gain energy and one must lose energy in order that the oscillations reach a steady sustained amplitude. If both $\alpha_{1}$ and $\alpha_{2}$ are negative, the system is absolutely stable; and if $\alpha_{1}$ and $\alpha_{2}$ are both positive, the motions grow without limit. The nonlinear terms in Eq. (45), and hence Eq. (47), cause transfer of energy from one mode to another.

Conditions for stability of the limit cycles are determined by examining the evolution of small disturbances from the stationary states. A set of inequalities is deduced from application of the Routh-Hurwitz or Lienard criteria to the characteristic polynomial. Figure 3 shows one way of displaying the results in terms of the linear parameters. Ranges of the parameters for which stable limit cycles exist are indicated by the hatched lines.

\section{B. Energy Transfer in Nonlinear Oscillations}

It is reasonable to associate with each mode an amount of energy $\varepsilon_{n}$ defined as the sum of kinetic and potential energies for the oscillator,

$$
\varepsilon_{n}=1 / 2\left(\dot{\eta}_{n}^{2}+\omega_{n}^{2} \eta_{n}\right)
$$

With the assumed form (32) for $\eta_{n}$, and the constraint (33), which implies

$$
\dot{\eta}_{n}=\omega_{n} \tau_{n}(t) \sin \left[\omega_{n} t+\phi_{n}(t)\right]
$$

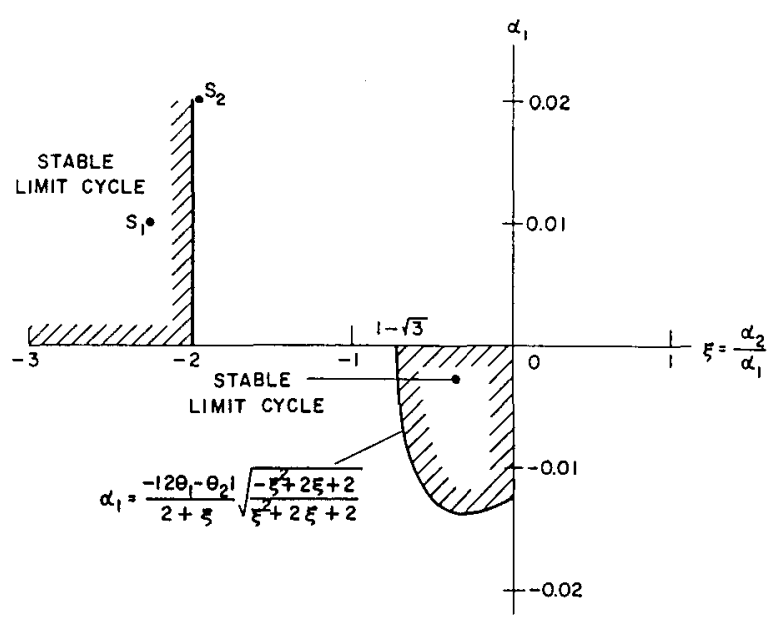

Fig. 3 Regions for stable limit cycles: two longitudinal modes, timeaveraged equations. 
we find $\mathscr{E}_{n}=1 / 2 \omega_{n} r_{n}^{2}$, with the assumptions involved in time averaging, this equals the time-averaged value,

$$
\left\langle\varepsilon_{n}\right\rangle=1 / 2 \omega_{n} r_{n}^{2}(t)
$$

where $r_{n}^{2}=A_{n}^{2}+B_{n}^{2}$. Now multiply Eqs. (47a-47d) by $A_{1}, B_{1}, B_{2}$, and add the results in pairs to form the equations for $r_{n}^{2}$

$$
\begin{aligned}
& \frac{1}{2} \frac{\mathrm{d} r_{1}^{2}}{\mathrm{~d} t}=\alpha_{1} r_{1}^{2}-\beta\left[A_{2}\left(A_{1}^{2}-B_{1}^{2}\right)+2 A_{1} B_{1} B_{2}\right] \\
& \frac{1}{2} \frac{\mathrm{d} r_{2}^{2}}{\mathrm{~d} t}=\alpha_{2} r_{2}^{2}+\beta\left[A_{1}\left(A_{1}^{2}-B_{1}^{2}\right)+2 A_{1} B_{1} B_{2}\right]
\end{aligned}
$$

The total time-averaged energy changes at the rate

$$
\frac{\mathrm{d}}{\mathrm{d} t}\langle\mathcal{E}\rangle=\frac{\mathrm{d}}{\mathrm{d} t}\left\langle\mathcal{E}_{1}\right\rangle+\frac{\mathrm{d}}{\mathrm{d} t}\left\langle\mathcal{\sigma}_{2}\right\rangle=\frac{1}{2} \omega_{1}^{2} \frac{\mathrm{d} r_{1}^{2}}{\mathrm{~d} t}+\frac{1}{2} \omega_{2}^{2} \frac{\mathrm{d} r_{2}^{2}}{\mathrm{~d} t}
$$

Substituting Eqs. (54a) and (54b) and setting $\omega_{2}=2 \omega_{1}$, we have

$$
\frac{1}{\omega_{1}^{2}} \frac{\mathrm{d}\langle\xi\rangle}{\mathrm{d} t}=\alpha_{1} r_{1}^{2}+4 \alpha_{2} r_{2}^{2}+3 \beta\left[A_{2}\left(A_{1}^{2}-B_{1}^{2}\right)+2 A_{1} B_{1} B_{2}\right]
$$

In the limit cycle, when the amplitudes are given by Eqs. (48), $\mathrm{d}\langle\delta\rangle / \mathrm{d} t=0$ because the amplitudes $r_{n}$ are independent of time: the right-hand sides of Eqs. (54a) and (54b) vanish. Note that the sum of Eqs. (54a) and (54b) gives $\alpha_{1} r_{1}^{2}+\alpha_{2} r_{2}^{2}$ for all time; the nonlinear terms cancel and $\alpha_{1} r_{1}^{2}+\alpha_{2} r_{2}^{2}$ represents the net rate at which energy flows to the system in the linear approximation. However, if we accept the definition (52), we find that the energy of the system changes due to the nonlinear dynamics, the second group of terms in Eq. (55). It is not presently clear what this result represents. We can also see its contribution to the behavior in the limit cycle by substituting Eq. (48) in Eq. (55) and keeping track of the terms; the result is

$$
\frac{\beta^{2}}{\omega_{1}^{2}} \frac{\mathrm{d}\langle\varepsilon\rangle}{\mathrm{d} t}=\underset{\substack{\text { Net linear gain/loss } \\ \text { of energy }}}{\alpha_{1}^{2} \alpha_{2}\left(1+\frac{\theta_{1}^{2}}{\alpha_{1}^{2}}\right)}-3 \alpha_{1}^{2} \alpha_{2}\left(1+\frac{\theta_{1}^{2}}{\alpha_{1}^{2}}\right)=0
$$

This result implies that there is no principle of detailed balancing for this system: the rate of energy loss from mode 1 due to the nonlinear processes is $\omega_{1}^{2} \alpha_{1}^{2} \alpha_{2}\left(1+\theta_{1}^{2} / \alpha_{1}^{2}\right) / \beta^{2}$ and energy is supplied to mode 2 by nonlinear processes four times as rapidly.

Thus, nonlinear processes cause energy to be transferred preferentially upward in the spectrum of modes, a phenomenon familiar as the steepening of a smooth compressive disturbance into a shock wave. For the acoustic systems treated with the formulation discussed here, that is a fundamental characteristic having much to do with observed nonlinear behavior.

\section{Nonlinear Transverse Modes}

It seems somewhat remarkable that simple conditions of existence and stability can be obtained for transverse modes, in a cylindrical chamber, by following the same approach used to treat longitudinal modes. The reason is that the time-averaged equations again have a special structure allowing construction of exact solutions for two modes. What makes this surprising is that because the natural frequencies do not satisfy the condition $\omega_{n}=n \omega_{1}$, the timeaveraged equations contain modulation on the right-hand side.
Before averaging, substitution of Eq. (37) in Eq. (35a) gives the set of equations valid for second-order acoustics,

$$
\begin{aligned}
& \frac{\mathrm{d} A_{n}}{\mathrm{~d} t}=-\frac{1}{2 \omega_{n}} \sum_{i=1}^{\infty}\left\{C_{n i}\left[\cos \left(\omega_{n}+\omega_{i}\right) t+\cos \left(\omega_{n}-\omega_{i}\right) t\right]\right. \\
& \left.+S_{n i}\left[\sin \left(\omega_{n}+\omega_{i}\right) t-\sin \left(\omega_{n}-\omega_{i}\right) t\right]\right\} \\
& \quad-\frac{1}{2 \omega_{n}} \sum_{i=1}^{\infty} \sum_{j=1}^{\infty}\left\{F _ { n i j } a _ { i j } \left[\cos \left(\omega_{n}+\omega_{i j+}\right) t\right.\right. \\
& \left.+\cos \left(\omega_{n}-\omega_{i j+}\right) t\right]+G_{n i j} b_{i j}\left[\cos \left(\omega_{n}+\omega_{i j+}\right) t\right. \\
& \left.+\cos \left(\omega_{n}-\omega_{i j+}\right) t\right]-F_{n i j} d_{i j}\left[\sin \left(\omega_{n}+\omega_{i j+}\right) t\right. \\
& \left.-\sin \left(\omega_{n}-\omega_{i j+}\right) t\right]+G_{n i j} e_{i j}\left[\sin \left(\omega_{n}+\omega_{i j-}\right) t\right. \\
& \left.\left.-\sin \left(\omega_{n}+\omega_{i j-}\right) t\right]\right\}
\end{aligned}
$$

where

$$
\begin{aligned}
\omega_{i j+}=\omega_{i}+\omega_{j} & \omega_{i j-}=\omega_{i}-\omega_{j} \\
C_{n i}=\omega_{i} D_{n i} A_{i}+E_{n i} B_{i} & S_{n i}=-\omega_{i} D_{n i} B_{i}+E_{n i} A_{i} \\
F_{n i j}=\omega_{i} \omega_{j} A_{n i j}-B_{n i j} & G_{n i j}=\omega_{i} \omega_{j} A_{n i j}+B_{n i j} \\
a_{i j}=1 / 2\left(A_{i} A_{j}-B_{i} B_{j}\right) & b_{i j}=1 / 2\left(A_{i} A_{j}+B_{i} B_{j}\right) \\
d_{i j}=1 / 2\left(A_{i} B_{j}+A_{j} B_{i}\right) & e_{i j}=1 / 2\left(A_{i} B_{j}-A_{j} B_{i}\right)
\end{aligned}
$$

A similar equation defines $\dot{B}_{n}$; see Yang and Culick $^{34}$ where further details of the analyses and the numerical values of the coefficients $F_{n i j}$ and $G_{n i j}$ are given. Three modes are considered: the first and second tangential and the first radial having the following wave numbers and mode shapes.

First tangential mode (1T):

$$
\kappa_{1} R=1.8412 \quad \psi_{1}=\cos \theta J_{1}\left(\kappa_{1} r\right) \quad \psi_{4}=\sin \theta J_{1}\left(\kappa_{1} r\right)
$$

First radial mode (1R):

$$
\kappa_{2} R=3.8317 \quad \psi_{2}=J_{0}\left(\kappa_{2} r\right)
$$

Second tangential mode (2T):

$$
\kappa_{3} R=3.0542 \quad \psi_{3}=\cos 2 \theta J_{2}\left(\kappa_{3} r\right) \quad \psi_{5}=\sin 2 \theta J_{2}\left(\kappa_{3} r\right)
$$

Degeneracy of the tangential modes leads to the existence of standing or spinning waves, but here we consider only standing waves.

One interesting feature is that the coefficients $F_{n n n}$ and $G_{n n n}$ associated with nonlinear gasdynamical self-coupling are nonzero only for the first radial mode. However, these are eliminated in the averaging process, and so, as in the case of purely longitudinal modes, there is apparently no significant self-coupling. Absence of self-coupling is a qualitative property of the problem that has significant consequences in respect to nonlinear stability.

\section{A. Time-Averaged Equations for Transverse Modes}

The averaged equations for $A_{n}$ and $B_{n}$ are as follows.

First tangential mode (1T):

$$
\begin{aligned}
& \frac{\mathrm{d} A_{1}}{\mathrm{~d} t}=\alpha_{1} A_{1}+\theta_{1} B_{1}+2\left[a_{1} b_{12} \cos \Omega_{1} t+a_{2} b_{13} \cos \Omega_{2} t\right] \\
& \quad+2\left[a_{1} a_{12} \sin \Omega_{1} t+a_{2} a_{13} \sin \Omega_{2} t\right]
\end{aligned}
$$




$$
\begin{aligned}
& \frac{\mathrm{d} B_{1}}{\mathrm{~d} t}=-\theta_{1} A_{1}+\alpha_{1} B_{1}-2\left[a_{1} b_{12} \sin \Omega_{1} t+a_{2} b_{13} \sin \Omega_{2} t\right] \\
& \quad+2\left[a_{1} a_{12} \cos \Omega_{1} t+a_{2} a_{13} \cos \Omega_{2} t\right]
\end{aligned}
$$

First radial mode (1R):

$$
\begin{aligned}
& \frac{\mathrm{d} A_{2}}{\mathrm{~d} t}=\alpha_{2} A_{2}+\theta_{2} \beta_{2}+b_{1} \\
& \quad \times\left[\left(A_{1}^{2}-B_{1}^{2}\right) \cos \Omega_{1} t-2 A_{1} B_{1} \sin \Omega_{1} t\right]
\end{aligned}
$$

$$
\begin{aligned}
& \frac{\mathrm{d} B_{2}}{\mathrm{~d} t}=-\theta_{2} A_{2}+\alpha_{2} B_{2}+b_{1} \\
& \quad \times\left[\left(A_{1}^{2}-B_{1}^{2}\right) \sin \Omega_{1} t+2 A_{1} B_{1} \cos \Omega_{1} t\right]
\end{aligned}
$$

Second tangential mode (2T):

$$
\begin{aligned}
& \frac{\mathrm{d} A_{3}}{\mathrm{~d} t}=\alpha_{3} A_{3}+\theta_{3} B_{3}+b_{2} \\
& \quad \times\left[\left(A_{1}^{2}-B_{1}^{2}\right) \cos \Omega_{2} t-2 A_{1} B_{1} \sin \Omega_{2} t\right] \\
& \frac{\mathrm{d} B_{3}}{\mathrm{~d} t}=\alpha_{3} B_{3}-\theta_{3} B_{3}+b_{2} \\
& \quad \times\left[\left(A_{1}^{2}-B_{1}^{2}\right) \sin \Omega_{2} t-2 A_{1} B_{1} \cos \Omega_{2} t\right]
\end{aligned}
$$

where

$$
\begin{gathered}
a_{1}=0.1570(\bar{a} / R) \quad a_{2}=-0.0521(\bar{a} / R) \\
b_{1}=0.1504(\bar{a} / R) \quad b_{2}=0.1873(\bar{a} / R) \\
\Omega_{1}=2 \omega_{1}-\omega_{2}=-0.1493(\bar{a} / R) \\
\Omega_{2}=2 \omega_{1}-\omega_{3}=-0.6282(\bar{a} / R)
\end{gathered}
$$

Note particularly that all of the nonlinear terms on the right-hand sides of Eqs. (61-65) contain modulation factors oscillating at either $\Omega_{1}$ or $\Omega_{2}$.

\section{B. Existence and Stability of Periodic Limit Cycles, Two Modes}

\section{First Tangential and First Radial Modes}

Write $A_{n}$ and $B_{n}$ in terms of an amplitude and phase

$$
A_{n}=r_{n}(t) \cos \Phi_{n}(t) \quad B_{n}=r_{n}(t) \sin \Phi_{n}(t)
$$

Substituting in Eqs. (60-63) and dropping terms dependent on the second tangential mode leads to the system

$$
\begin{aligned}
& \frac{\mathrm{d} r_{1}}{\mathrm{~d} t}=\alpha_{1} r_{1}+a_{1} r_{1} r_{2} \cos \left(2 \Phi_{1}-\Phi_{2}+\Omega_{1} t\right) \\
& \frac{\mathrm{d} r_{2}}{\mathrm{~d} t}=\alpha_{2} r_{2}+b_{1} r_{1}^{2} \cos \left(2 \Phi_{1}-\Phi_{2}+\Omega_{1} t\right) \\
& \frac{\mathrm{d} \Phi_{1}}{\mathrm{~d} t}=-\theta_{1}-a_{1} r_{2} \sin \left(2 \Phi_{1}-\Phi_{2}+\Omega_{1} t\right)
\end{aligned}
$$

$$
\frac{\mathrm{d} \Phi_{2}}{\mathrm{~d} t}=-\theta_{2}+b_{1}\left(r_{1}^{2} / r_{2}\right) \sin \left(2 \Phi_{1}-\Phi_{2}+\Omega_{1} t\right)
$$

The fact that all of the right-hand sides contain the same timevarying argument, $2 \Phi_{1}-\Phi_{2}+\Omega_{1} t$ is crucial to producing simple results.

In the limit cycle, the amplitudes $r_{n 0}$ are constant, and their values are found as the solutions to the algebraic equations given by setting to zero the left-hand sides of Eqs. (68-71). The results are

$$
\begin{aligned}
& r_{10}=\sqrt{\frac{\alpha_{1} \alpha_{2}}{a_{1} b_{1}}}\left[1+\left(\frac{2 \theta_{1}-\theta_{2}-\Omega_{1}}{2 \alpha_{1}+\alpha_{2}}\right)^{2}\right]^{1 / 2} \\
& r_{20}=-\frac{\alpha_{1}}{a_{1}}\left[1+\left(\frac{2 \theta_{1}-\theta_{2}-\Omega_{1}}{2 \alpha_{1}+\alpha_{2}}\right)^{2}\right]^{1 / 2}
\end{aligned}
$$

Because $a_{1} b_{1}$ is negative (see the values given earlier), $\alpha_{1} \alpha_{2}$ must be negative to make $r_{10}$ real. The physical reason is the same as that explained in connection with the same result [Eq. (51)] for longitudinal modes.

Just as for longitudinal modes, we must allow for frequency shifts in the limit cycle. Eventually the results are found for the time-dependent amplitudes $\eta_{n 0}$

$$
\begin{aligned}
& \eta_{10}=r_{10} \sin \left[\left(\omega_{1}+v_{1}\right) t+\zeta_{1}\right] \\
& \eta_{20}=r_{20} \sin \left[2\left(\omega_{1}+v_{1}\right) t+\zeta_{2}\right]
\end{aligned}
$$

where

$$
2 \zeta_{1}-\zeta_{2}=\tan ^{-1}\left[\frac{\left(\omega_{2}-\theta_{2}\right)-2\left(\omega_{1}-\theta_{1}\right)}{2 \alpha_{1}+\alpha_{2}}\right]
$$

We can set either $\zeta_{1}$ or $\zeta_{2}$ equal to zero because zero phase is arbitrary. In the limit cycle, the participating motions must have frequencies that are integral multiples of the fundamental in order that the motion be periodic. That Eqs. (74a) and (74b) satisfy this requirement is a partial confirmation that the approximations used in the averaging process are correct. The result was found by Zinn and Powell ${ }^{13}$ as well in their numerical analysis of transverse modes. Figure 4 shows two examples of limit cycles for this case; the values of the limiting amplitudes are independent of the initial conditions.

Establishing the conditions for stability of the limit cycles follows the procedure outlined in the preceding section. The necessary and sufficient conditions are

$$
\begin{gathered}
\alpha_{1} \alpha_{2}<0 \\
\alpha_{1}+\alpha_{2}<0 \\
2 \alpha_{1}+\alpha_{2}<0
\end{gathered}
$$

For $\alpha_{1}<0$ the additional condition must be met

$$
\alpha_{1}>\frac{-|K|}{2+\xi}\left[\frac{-\xi^{2}+2 \xi+2}{\xi^{2}+2 \xi+2}\right]^{1 / 2}
$$

where $K=2 \theta_{1}-\theta_{2}-\Omega_{1}$ and $\xi=\alpha_{2} / \alpha_{1}$ must lie in the range 1 $-\sqrt{3}<\xi<0$. Figure 5 shows the regions of $\alpha_{1}$ and $\alpha_{2} / \alpha_{1}$, for $K$ $=0.25$, in which the limit cycles are stable.

\section{First and Second Tangential Modes}

Because Eqs. (64) and (65) are Eqs. (62) and (63) with ()$_{2} \rightarrow$ ()$_{3}$, the equations for the amplitudes and phases in this case have exactly the same form as Eqs. (68-71), except that dependence on 

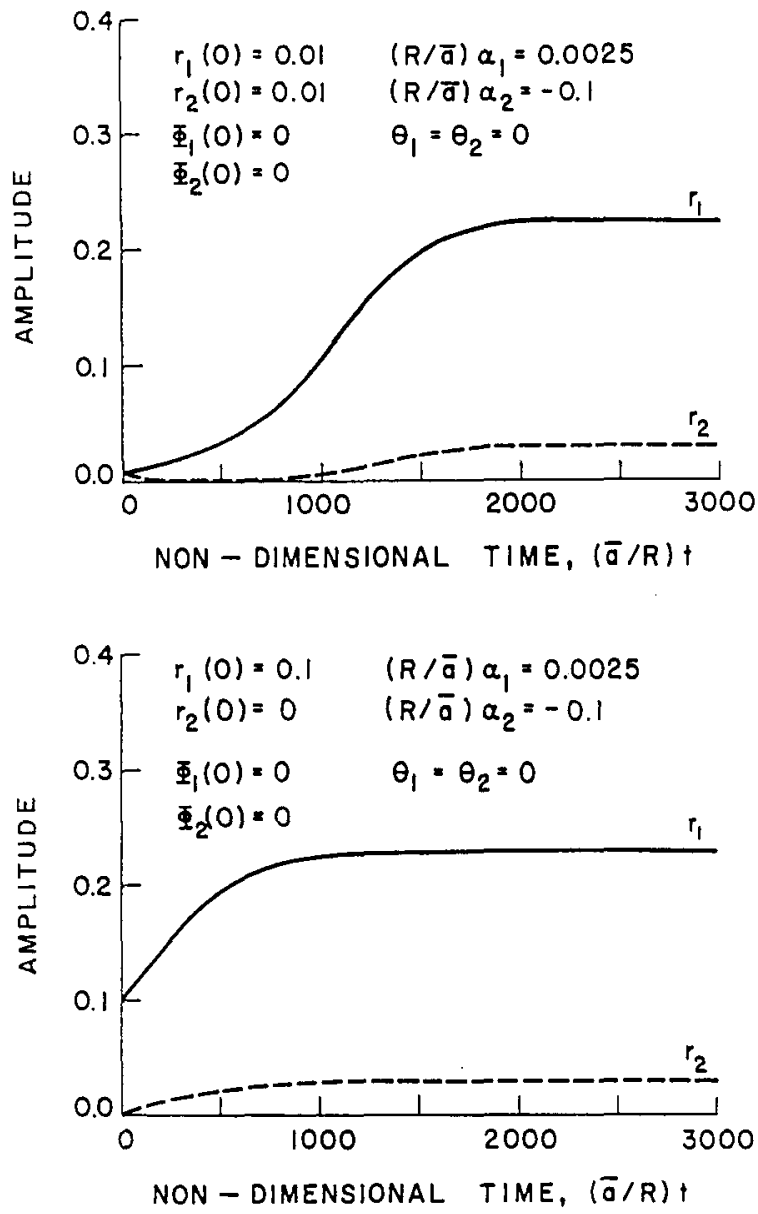

Fig. 4 Amplitudes in limit cycles: first tangential and first radial modes, time-averaged equations.

the radial mode is replaced by dependence on the second tangential mode. The results for existence and stability, therefore, are the same as well; in particular, Fig. 6 applies with a reinterpretation of the symbols. However, the difference between the frequencies of the first radial and second tangential modes produces differences in the quantitative behavior of the limit cycles.

The point is best made by comparing results obtained for the two cases. Figure 6 shows the evolution of the amplitudes for coupled 1T/2T modes for the same values of initial conditions and linear parameters used for the upper result in Fig. 4 (note that $\theta_{1}=\theta_{2}$ $=0$ in these examples). Now the amplitudes are substantially greater because $\Omega_{2}>\Omega_{1}$ and $a_{2} b_{2}<a_{1} b_{1}$. In fact, the amplitudes in Fig. 3 are well outside the range for which this analysis is valid. $A$ lower value of $\alpha_{1}$ will produce lower amplitudes.

Considerations of the energy flow in this system ${ }^{34}$ suggest reasons why the differences between the 1T/1R and 1T/2T cases are large, but the matter is not yet satisfactorily explained. Following the ideas introduced in Sec. V.B, we define the energy associated with each mode as the sum of kinetic and potential energy of the associated oscillator, $\mathcal{E}_{n}=\left(\bar{\eta}_{n}^{2}+\omega_{n}^{2} \eta_{n}^{2}\right) / 2$, then we can use the averaged equations to find equations for the time evolution of the average energy in each mode, corresponding to Eqs. (54a) and (54b) for longitudinal modes.

1T mode:

$$
\frac{\mathrm{d}\left\langle\tilde{\sigma}_{1}\right\rangle}{\mathrm{d} t}=\alpha_{1} \omega_{1}^{2} r_{1}^{2}+a_{1} \omega_{1}^{2} r_{1}^{2} r_{2} \cos \left[\Omega_{1} t+2 \Phi_{1}-\Phi_{2}\right]
$$

1R mode:

$$
\frac{\mathrm{d}\left\langle\varepsilon_{2}\right\rangle}{\mathrm{d} t}=\alpha_{2} \omega_{2}^{2} r_{2}^{2}+b_{1} \omega_{2}^{2} r_{1}^{2} r_{2} \cos \left[\Omega_{1} t+2 \Phi_{1}-\Phi_{2}\right]
$$

2T mode:

$$
\frac{\mathrm{d}\left\langle\mathcal{G}_{3}\right\rangle}{\mathrm{d} t}=\alpha_{3} \omega_{3}^{2} r_{3}^{2}+b_{2} \omega_{3}^{2} r_{1}^{2} r_{3} \cos \left[\Omega_{2} t+2 \Phi_{1}-\Phi_{3}\right]
$$

By summing the appropriate pairs chosen from these equations, we form the equation for the total rate of energy change.

1T/1R:

$$
\begin{aligned}
& \frac{\mathrm{d}\langle\tilde{G}\rangle}{\mathrm{d} t}=2 \alpha_{1}\left\langle\mathscr{E}_{1}\right\rangle+2 \alpha_{2}\left\langle\mathscr{E}_{2}\right\rangle \\
& \quad+\left(\omega_{1}^{2} a_{2}+\omega_{2}^{2} b_{1}\right) r_{1}^{2} r_{2} \cos \left(\Omega_{1} t+2 \Omega_{1}-\Omega_{2}\right)
\end{aligned}
$$

1T/2T:

$$
\begin{aligned}
& \frac{\mathrm{d}\langle\mathcal{G}\rangle}{\mathrm{d} t}=2 \alpha_{1}\left\langle\mathcal{E}_{1}\right\rangle+2 \alpha_{3}\left\langle\mathcal{E}_{3}\right\rangle \\
& \quad+\left(\omega_{1}^{2} \alpha_{1}+\omega_{2}^{2} b_{2}\right) r_{1}^{2} r_{3} \cos \left(\Omega_{2} t+2 \Phi_{1}-\Phi_{3}\right)
\end{aligned}
$$

Comparison of the two equations [(79) and (80)], with Eq. (55) for longitudinal modes shows again the presence of contributions from nonlinear processes to the evolution of the total energy. Once again, we find that in the limit cycles the averaged energy is constant, but not due to a simple balance of the energy gains and losses associated only with linear processes. We should note that these nonlinear terms do not represent internal dissipation of energy, for they do not produce entropy.

The additional terms in question have quite different numerical values for the $1 T / 1 R$ and $1 T / 2 T$ problems. The rate of energy extraction for the 1T mode, due to the nonlinear processes, is smaller in the second case than in the first. As a result, for the same linear contributions $\left(\alpha_{1}, \alpha_{2}\right)$, the amplitudes in the limit cycle are higher for the 1T/2T system than for the 1T/1R system.

\section{Periodic Limit Cycles for Three Modes}

Results have been obtained ${ }^{34}$ for the case when all three of the lowest transverse modes (1T/1R/2T) are accounted for.

The amplitudes in the limit cycle are

$$
\begin{gathered}
r_{10}=1 /\left[\left(\frac{a_{1} b_{1}}{\alpha_{1} \alpha_{1}} \cos ^{2} X_{0} \frac{a_{2} b_{2}}{\alpha_{1} \alpha_{3}} \cos ^{2} Y_{0}\right)^{1 / 2}\right] \\
r_{20}=-\left(b_{1} / \alpha_{2}\right) r_{10}^{2} \cos X_{0} \\
r_{30}=-\left(b_{2} / \alpha_{3}\right) r_{10} \cos Y_{0} \\
X_{0}=2 \zeta_{1}-\zeta_{2} \quad Y_{0}=2 \zeta_{1}-\zeta_{3}
\end{gathered}
$$

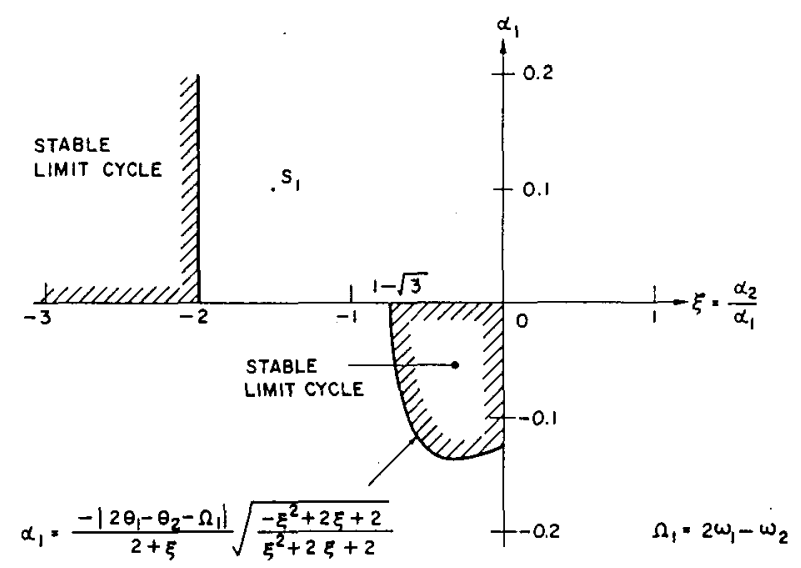

Fig. 5 Regions for stable limit cycles: first tangential and first radis: modes, time-averaged equations. 
and $\zeta_{1}, \zeta_{2}, \zeta_{3}$ are constants determined as part of the analysis. Simple closed-form results cannot be obtained, although some inequalities exist setting limits on the allowable ranges of parameters for existence. No simple statements can be made about stability.

Numerical results show when the $1 \mathrm{R}$ mode is accounted for, the amplitudes of the $1 \mathrm{~T}$ and $2 \mathrm{~T}$ modes are greatly reduced from those found in the two-mode approximation, 1T/2T. Perhaps the most important implication of this conclusion is that one should probably be wary of a two-mode approximation for transverse modes: modal truncation may bring about serious errors not yet understood.

\section{Nonlinear Instability, Triggering, and Third- Order Acoustics}

There are chiefly two classes of nonlinear problems dominating both practical and theoretical work on combustion instabilities: the conditions for existence and stability of limit cycles and the conditions under which a linear system may be unstable to a sufficiently large disturbance. We have examined the first class in the preceding two sections and we turn now to the second, which includes the phenomenon called triggering. By triggering we shall mean that a linearly stable system may be caused to execute stable limit cycles. It is quite possible also that large disturbances may produce unstable motions that grow without limit according to the analysis. Such a result would suggest that the physical model used as the basis for the calculations may be deficient. For example, higher order nonlinearities might cause the limit cycle to be stable. In any case, as a convenience here, we use the term triggering in the restricted sense of pulsing to stable limit cycles, where in most of the literature the term refers to pulsing a linearly stable system to stable or unstable limit cycles.

For applications, it is most important to be able to understand and predict the amplitudes of motions in a limit cycle; in practice one would generally like to have zero amplitudes always, or at least know how to achieve that condition. The results obtained so far for both cases of longitudinal and transverse modes showed that, for second-order gasdynamical nonlinearities, the limit cycles of a linearly unstable system are unique. That is, except for possible ambiguities of phase, the motions in the limit cycle are independent of the initial conditions. No exceptions have been found in all of the numerical results carried out to date.

However, there is much data showing that many laboratory devices and full-scale systems that are linearly stable can be driven into unstable motions and limit cycles if a sufficiently large disturbance is introduced. This phenomenon is the basis for assessing the stability of propulsion systems, particularly liquid rockets ${ }^{1}$ and more recently solid rockets. ${ }^{4}$ Moreover, it is possible that large unwanted disturbances can be generated during operations if pieces of solid material pass through the exhaust nozzle, or if there are regions of rapid buming (such as accumulation of liquid reactants on walls, or burning in cracks in a solid propellant).

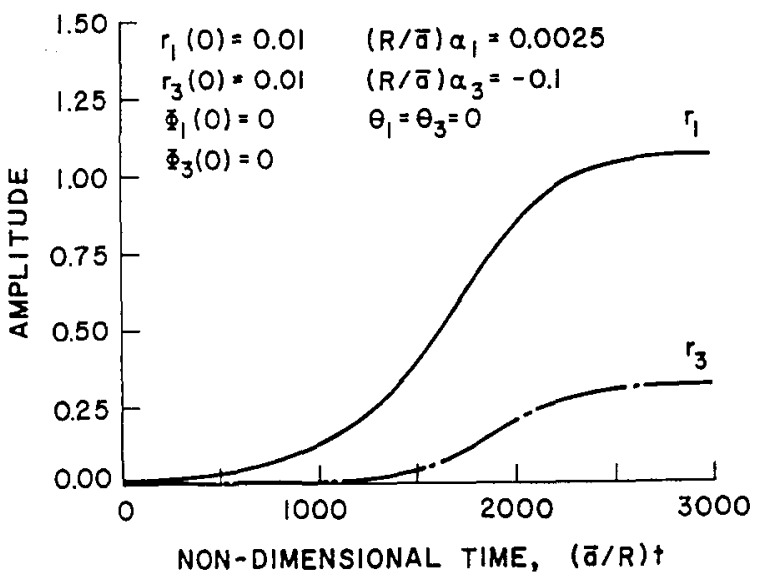

Fig. 6 Amplitudes in a limit cycle: first and second tangential modes, time-averaged equations.
Thus the predictions of the approximate analysis discussed so far are not consistent with observations of triggering and more recently solid rockets, ${ }^{4}$ although there is reason to believe that satisfactory results can be obtained for the nonlinear behavior of linearly unstable systems over broad ranges of conditions. One immediate way of resolving the differences is to incorporate a nonlinear representation for the combustion processes. For example, setting a threshold of amplitude, below which all $\alpha_{n}$ are negative and above which one or more are positive, introduces the opportunity for triggering. This possibility arises in particular from "velocity coupling" when the interaction between combustion processes depends on the magnitude of the velocity parallel to a burning solid surface. Numerical analysis (e.g., Baum et al. ${ }^{2,4}$ ) has confirmed these expectations.

Here we shall confine our attention to the possible consequences of gasdynamic nonlinearities and coupling between unsteady motions and the mean velocity field. There is considerable previous work on this aspect of the subject, largely based on numerical solutions to the partial differential equations of conservation. It is difficult to perceive systematic trends in the results, and few general conclusions can be drawn. See Yang et al. ${ }^{35}$ for a brief review of earlier work and for a more thorough treatment of the results discussed here.

Initially, the work described here was motivated by the idea that whereas second-order acoustics would seem not to contain triggering, there is reason to anticipate that third-order acoustics would. The basis for that notion is the well-known behavior of simple one-dimensional systems described by the equation

$$
\frac{\mathrm{d} r}{\mathrm{~d} t}=\alpha r+b r^{2}+c r^{3}=k r\left(r_{1}-r\right)\left(r_{2}-r\right)
$$

Figure 4 shows that for $\alpha$ negative (the system is linearly stable) the possibility exists for triggering to a stable limit cycle, amplitude $r_{2}$, if the initial disturbance has amplitude greater than $r_{1}$. The second-order form of Eq. (82) does not contain triggering to a stable limit cycle; an initial disturbance larger than $r_{1}$ (if the factor $r_{2}-r$ is dropped) will produce an unstable motion.

Following earlier work by Awad, ${ }^{36}$ Yang et al. ${ }^{35}$ have discussed several model problems of this sort to try to clarify, or at least to suggest, the prospects for defining general conditions for triggering. They examined one- and two-dimensional models. Their conclusions are only modestly helpful for understanding the acoustics problem because it appears that the nonlinear behavior is sensitive both to the number of degrees of freedom and to the structure of the nonlinear coupling between the degrees of freedom, i.e. between the model oscillators.

In particular, the distinction between "self-coupling" and "cross-coupling" is especially significant. Self-coupling refers to terms in the equation for $r_{n}$, say, which are nonlinear in $r_{n}$ only $\left(r_{n}^{2}, r_{n}^{3}, \ldots,\right)$; thus Eq. (82) contains only self-coupling. Crosscoupling refers to nonlinear coupling between modes. The model problems suggest that triggering is greatly encouraged by self-coupling as, for example, Fig. 7 based on Eq. (82) suggests. This conclusion evidently remains true for cases of several degrees of freedom, which is interesting because the second-order acoustics equations containing gasdynamic nonlinearities only do not have self-coupling terms, although they almost do: equations for $A_{n}$ may contain terms in $B_{n}^{2}$ and vice versa. The point is that the general structure of Eqs. (35a) and (35b) with $F_{n}$ given by Eq. (37) evidently do not contain triggering, at least for most values of the linear parameters.

The importance of self-coupling is suggested by the following model problem for two amplitudes:

$$
\frac{\mathrm{d} r_{1}}{\mathrm{~d} t}=\alpha_{1} r_{1}+b_{1} r_{2}+c_{1} r_{1}^{2} \quad \frac{\mathrm{d} r_{2}}{\mathrm{~d} t}=\alpha_{2} r_{2}+b_{2} r_{1}^{2}
$$

The conditions for the existence and stability of limit cycles are quite easy to derive, using the methods outlined earlier. ${ }^{35}$ For $c_{1}$ $=0$ triggering to a stable limit cycle does not exist (although large 


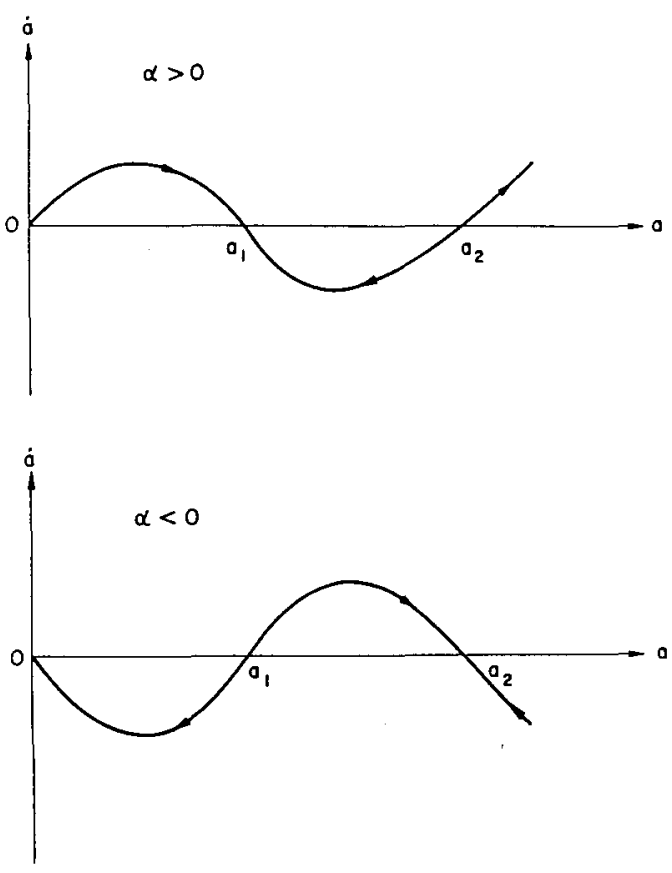

Fig. 7 Phase plane for a second-order model system having self-coupling.

disturbances can destabilize a linearly stable system, the motions grow without limit). However, for $c_{1} \neq 0$, it is possible to trigger stable limit cycles, an example of which is shown in Fig. 8.

To represent triggering to stable limit cycles within the framework of the approximate analysis discussed here, it appears that there are three possible extensions of the formulation containing only second-order gasdynamical nonlinearities: 1) incorporate qualitatively different nonlinear processes, nonlinear combustion being the most attractive; 2) account for higher order gasdynamic nonlinearities; and 3) include nonlinear interactions between the mean flowfield and the unsteady motions. Modal truncation seems not to be an influence on triggering, but the possible effects of time averaging remain unclear.

We shall have relatively little to say about nonlinear combustion in this paper, although numerical analyses ${ }^{2,4,32,37}$ have shown that nonlinear transient burning and velocity coupling are crucial to explaining nonlinear behavior observed in laboratory tests of solid propellant rocket motors. Figure 9, taken from the careful summary of the subject by Baum et al., ${ }^{4}$ shows a good comparison of measured and computed results.

Much less has been done with nonlinear combustion modeling in the approximate analysis. Awad ${ }^{36}$ and Yang et al. ${ }^{38}$ have examined some model examples, providing a useful beginning, but much remains to be done; work on this subject is in progress. Although it is relatively easy to account for nonlinear combustion processes, it is difficult to confim that a particular model is realistic. Part of the reason for this difficulty is that fairly realistic results can be obtained with different models. It is not easy to establish uniqueness.

Thus it has seemed more important at this stage to investigate the consequences of nonlinear processes whose structure is much better known: items 2 and 3 of the possible extensions cited earlier. In fact, only a small part of the possible classes of problems has been examined. The richness of possibilities is due to the fact that we are dealing with a physical situation characterized by two small parameters: reference Mach numbers $\bar{M}_{0}$ and $M_{0}^{\prime}$ of the mean and unsteady flows. Lowest order problems are readily formulated: linear stability, the limit of vanishingly small $\bar{M}_{0}$, and $M_{0}^{\prime}$, with $M_{0}^{\prime}$ « $\bar{M}_{0}$; and nonlinear second-order acoustics, for which $M_{0}$ and $M_{0}^{\prime}$ are of the same order in the limit $\bar{M}_{0}, M_{0}^{\prime} \rightarrow 0$. When one proceeds to higher order, there is no rule for setting rigorously the orders of terms to be retained. Thus arbitrary choices must be made. Investigations to date have involved either acoustics nonlin- earities of third order with no dependence on the mean flow (item 2) or contributions of second order in the acoustic fluctuations and first order in the mean flow (item 3). We shall briefly summarize some of the results in Secs. VII.A and VII.B.

\section{A. Third-Order Acoustics}

$\mathrm{Kim}^{39}$ and Yang et al..$^{38,40}$ have treated third-order acoustics as a direct extension of the second-order acoustics discussed already. We have already noted that, based on the behavior of the onedimensional model described by Eq. (82) and Fig. 4, one might expect that third-order acoustics would contain triggering to stable limit cycles. That turns out not to be true, a conclusion apparently consistent with the results of numerical analyses. The chief reason seems to be that the nonlinear coupling causes energy transfer between modes such that the energy of an initial pulse is either ultimately dissipated (so the motion dies out) or the transfer into one or more modes is so concentrated that an unstable motion is produced. Without the presence of self-coupling, there is evidently no process to balance the transfer of energy so as to cause a steady limit cycle. The physical interpretation is not thoroughly understood. However, the essential reasons must be related to the special structure of the gasdynamic nonlinearities.

The problem of determining the conditions under which triggering to stable limit cycles exists remains unsolved. Conclusions reached with the approximate analysis described here do not seem to be entirely consistent with those reported in earlier works cited in Sec. III. Because the methods used are quite different, and because the $n-\tau$ combustion model has not been thoroughly studied in the present work, it is not possible to identify the precise reasons for the differences in the results. It is a fundamental matter that must eventually be solved.

Before summarizing the evidence supporting those conclusions, we should cite an interesting computation of nonlinear behavior, by Flandro, ${ }^{41}$ to try to explain limit cycles and triggering for longitudinal waves. The work was motivated by extensive observations made during an experimental program carried out over several years. ${ }^{42}$ By considering only the energy expressed to third order in the pressure and velocity fluctuations, Flandro derived an equation for the rate of change of $\varepsilon$, which he calls the "composite amplitude,"

$$
\frac{\mathrm{d} \varepsilon}{\mathrm{d} t}=A \varepsilon+B \varepsilon^{2}+C \varepsilon^{3}
$$

This equation has the same form as Eq. (82) and, therefore, if the coefficients have values in appropriate ranges, it can be used to predict triggering to stable limit cycles.

Flandro demonstrated good qualitative agreement between solutions to the preceding equation and measurements of the decay of large amplitude pulses. Moreover, he was able to find fairly good approximations to the amplitudes in limit cycles and levels of dis-

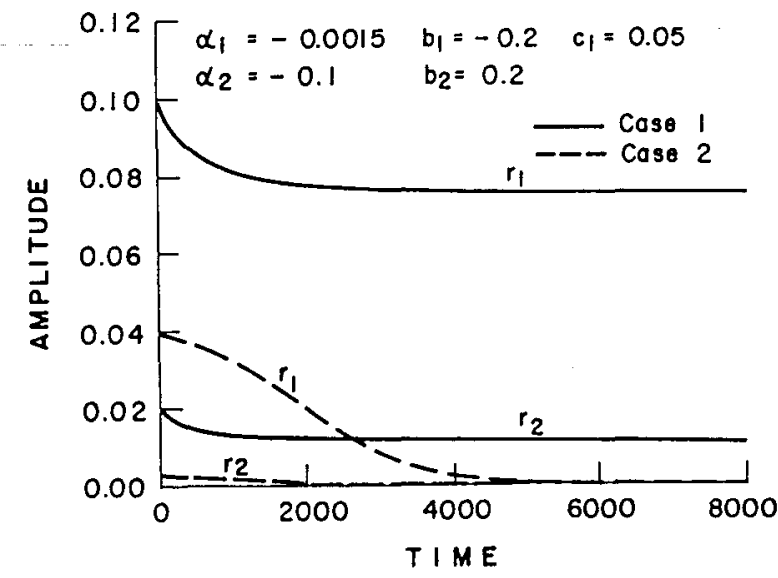

Fig. 8 Example of triggering to a stable limit cycle for a system of two modes containing self-coupling. 
turbances required to produce triggering in small solid propellant rockets. The dependence of the coefficients $A, B$, and $C$ on parameters characterizing the motors suggests possible trends useful in design and correlating data.

That analysis, and the nature of the results, are appealing and evidently possess some measure of reality. One must conclude that, contrary to the conclusion reached in numerical analysis and in other approximate analyses cited here and discussed subsequently, third-order acoustics does indeed contain an explanation for triggering to stable limit cycles, and under quite broad practical conditions. How are these two contradictory conclusions to be reconciled? The answer seems to lie in Flandro's definition and treatment of the composite amplitude $\varepsilon$. Initially, $\varepsilon$ is introduced as a small parameter measuring the pressure amplitude $p=\bar{p}+\varepsilon p^{\prime}$. All other flow variables are assumed to be measureable by $\varepsilon$ as well and are expanded in power series as $\rho=\bar{\rho}+\varepsilon \rho_{1}+\varepsilon^{2} \rho_{2}+\cdots+, u$ $=\bar{u}+\varepsilon u_{1}+\varepsilon^{2} u_{2}+\cdots+$, etc. Hence, the acoustic energy itself turns out to be expressible as $\mathcal{E}=\varepsilon^{2} \mathcal{E}_{1}+\varepsilon^{3} \mathcal{E}_{2}+\cdots+$.

Then Flandro makes the basic and crucial assumption that all of the time dependence of the fluctuating quantities is contained in the single quantity $\varepsilon$. This implies, for example, that if the pressure fluctuation $\varepsilon p^{\prime}$ is analyzed as a Fourier decomposition, all modes must exhibit exactly the same time dependence. Both theory and experiment show that this is incorrect. The consequences of the assumption are considerable: if the transient motions of the system are incorrectly modeled, then because nonlinear behavior is commonly dependent on its history in many respects, one is then on weak ground using a single quantity $\varepsilon$ and its time evolution as the basis for explaining the behavior of systems having many degrees of freedom.

On the other hand, Flandro's approach should not be dismissed out of hand. The ideas he has treated are an important part of the picture, and some of his predictions are persuasively realistic. It is possible that his model of the behavior, or a modified form of it, could be given a more rigorous foundation, thereby providing a more satisfactory reconciliation of his analysis with other works.

When the expansion is carried out to third order and coupling between the mean flow and nonlinear acoustical motions is ignored, the force $F_{n}$ defined by Eq. (31) has the form

$$
\begin{aligned}
& F_{n}=-\sum^{i}\left[D_{n i} \dot{\eta}_{i}+E_{n i} \eta_{i}\right]-\sum_{i=1}^{\infty} \sum_{j=1}^{\infty}\left[A_{n i j} \dot{\eta}_{i} \dot{\eta}_{j}+B_{n i j} \eta_{i} \eta_{n}\right] \\
& -\sum_{i=1}^{\infty} \sum_{j=1}^{\infty} \sum_{m=1}^{\infty}\left[R_{n i j m} \dot{\eta}_{i} \dot{\eta}_{j} \eta_{m}+S_{n i j m} \eta_{i} \eta_{j} \eta_{m}\right]
\end{aligned}
$$

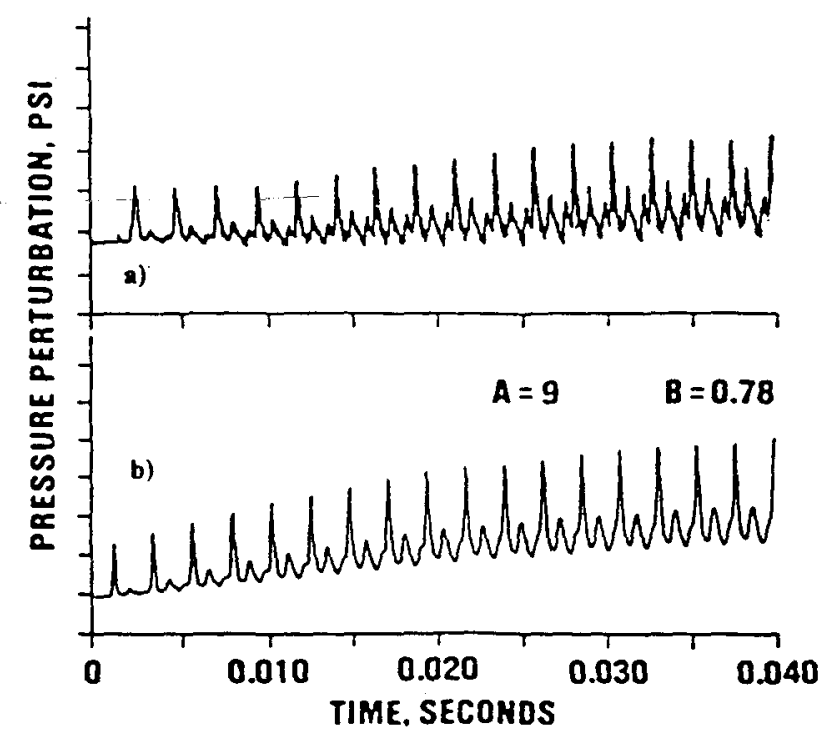

Fig. 9 Comparison of computed and measured behavior subsequent to an initial pulse.
This result is, of course, still valid for any geometry, i.e., any modal system, as long as the modes or basis functions $\psi_{n}$ are orthogonal.

\section{Longitudinal Modes}

For purely longitudinal modes, the double and triple sums become single and double sums, respectively. Exact results for third-order acoustics have been obtained only for two modes, for which the equations are

$$
\begin{aligned}
& \frac{\mathrm{d} A_{1}}{\mathrm{~d} t}=\alpha_{1} A_{1}+\theta_{1} B_{1}-\beta\left(A_{1} A_{2}+B_{1} B_{2}\right) \\
& +\beta_{1}\left(A_{1}^{2}+B_{1}^{2}\right) B_{1}-\beta_{2}\left(A_{2}^{2}+B_{2}^{2}\right) B_{1} \\
& \frac{\mathrm{d} B_{1}}{\mathrm{~d} t}=\alpha_{1} B_{1}-\theta_{1} A_{1}-\beta\left(A_{1} B_{2}-A_{2} B_{1}\right) \\
& -\beta_{1}\left(A_{1}^{2}+B_{1}^{2}\right) A_{1}+\beta_{2}\left(A_{2}^{2}+B_{2}^{2}\right) A_{1} \\
& \frac{\mathrm{d} A_{2}}{\mathrm{~d} t}=\alpha_{2} A_{2}+\theta_{2} B_{2}+\beta\left(A_{1}^{2}+B_{1}^{2}\right) \\
& \quad+2 \beta_{1}\left(A_{2}^{2}+B_{2}^{2}\right) B_{2}-2 \beta_{1}\left(A_{1}^{2}+B_{1}^{2}\right) B_{2} \\
& \frac{\mathrm{d} B_{2}}{\mathrm{~d} t}=\alpha_{2} B_{2}-\theta_{2} A_{2}+2 \beta A_{1} B_{1} \\
& -2 \beta_{1}\left(A_{2}^{2}+B_{2}^{2}\right) A_{2}+2 \beta_{2}\left(A_{1}^{2}+B_{1}^{2}\right) A_{2}
\end{aligned}
$$

where the two new constants associated with the third-order acoustic coupling are

$$
\beta_{1}=\frac{5-3 \bar{\gamma}}{64 \bar{\gamma}^{2}} \omega_{1} \quad \beta_{2}=\frac{\bar{\gamma}-1}{16 \bar{\gamma}^{2}} \omega_{1}
$$

Written in terms of amplitudes and phases, Eqs. (85a-85d) are

$$
\begin{gathered}
\frac{\mathrm{d} r_{1}}{\mathrm{~d} t}=\alpha_{1} r_{1}-\beta r_{1} r_{2} \cos \psi \\
\frac{\mathrm{d} r_{2}}{\mathrm{~d} t}=\alpha_{2} r_{2}+\beta r_{1}^{2} \cos \psi \\
\frac{\mathrm{d} \psi}{\mathrm{d} t}=-2 \theta_{1}+\theta_{2}-\beta\left(\frac{r_{1}^{2}}{r_{2}}-2 r_{2}\right) \sin \psi+2\left(\beta_{1}+\beta_{2}\right)\left(r_{2}^{2}-r_{1}^{2}\right)
\end{gathered}
$$

with

$$
\psi=2 \Phi_{1}-\Phi_{2}
$$

Note that the third-order coupling does not appear in the equations for the amplitude, a curious result reflecting the special, and in a certain sense limited, character of the gasdynamical coupling. The energy transfer between modes does affect the phase difference.

As in the case of second-order acoustics, the nice practical advantage of considering only two modes is that literal conditions 
for existence and stability can be established. The conditions for existence are

$$
\begin{gathered}
\alpha_{1} \alpha_{2}<0 \\
a_{1}^{2}-4 a_{3}\left(\alpha_{2}+\alpha_{3}\right)>0
\end{gathered}
$$

where

$$
\begin{gathered}
a_{1}=\left(2 a_{1}+a_{2}\right) \beta^{2} \\
a_{2}=\left(-2 \theta_{1}+\theta_{2}\right) \beta^{2} \\
a_{3}=2\left(\beta_{1}+\beta_{2}\right) \alpha_{1}\left(\alpha_{1}+\alpha_{2}\right)
\end{gathered}
$$

The amplitudes and phase in the limit cycle are

$$
\begin{gathered}
r_{10}=\frac{\sqrt{-\alpha_{1} \alpha_{2}}}{\beta \cos \Psi_{0}} \\
r_{20}=\frac{\alpha_{1}}{\beta \cos \Psi_{0}} \\
\cos ^{2} \Psi_{0}=\frac{1}{2\left(a_{1}^{2}+a_{2}^{2}\right)}\left[a_{1}^{2}-2 a_{2} a_{3} \pm a_{1} \sqrt{a_{1}^{2}-4 a_{2} a_{3}-4 a_{3}^{2}}\right]
\end{gathered}
$$
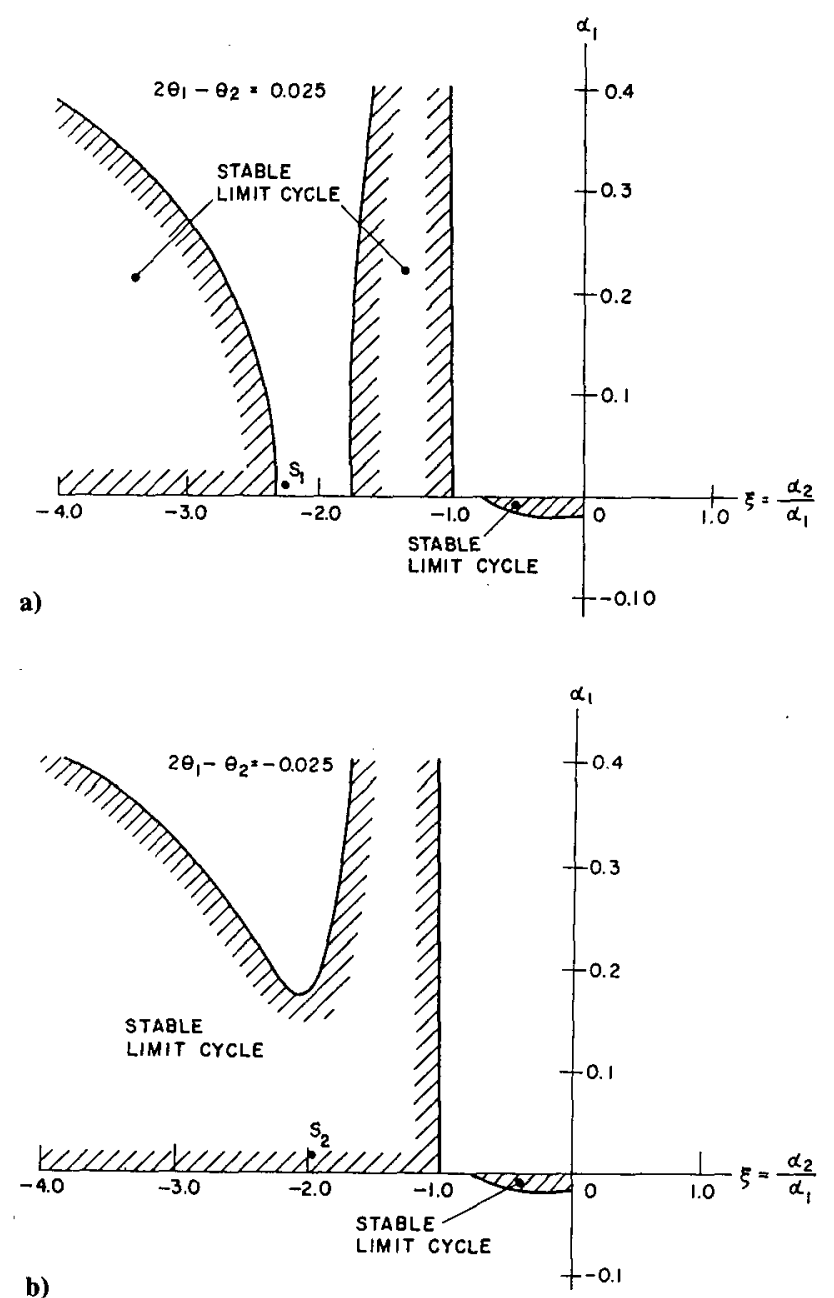

b)

Fig. 10 Regions for stable limit cycles: two longitudinal modes with third-order acoustics, time-averaged equations.

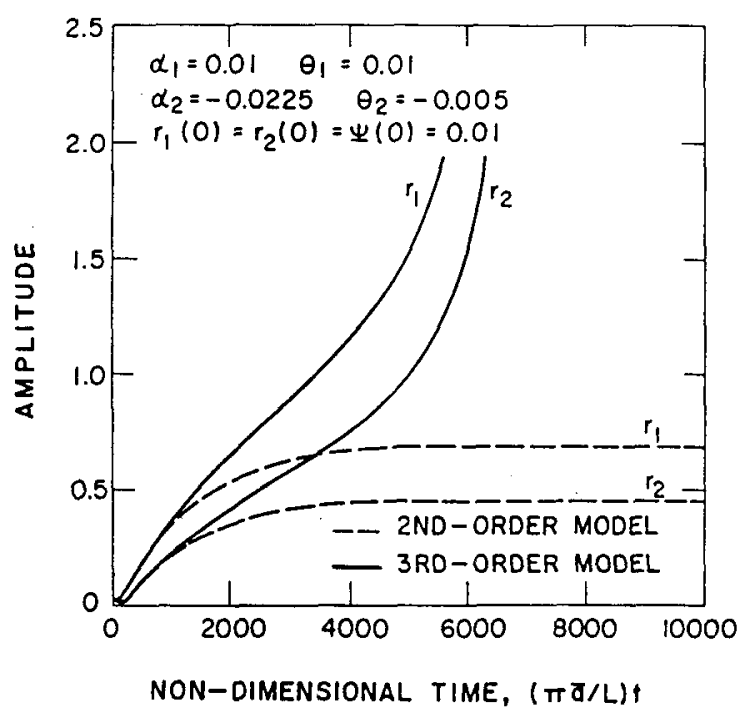

Fig. 11 Example showing destabilizing effects of third-order acoustics: two longitudinal modes, time-averaged equations.

Necessary and sufficient conditions for stability of the limits are given in Refs. 38-40. Figure 10 gives one illustration of the dependence of stable limit cycles on the linear parameters $\left(\alpha_{n}, \theta_{n}\right)$.

These results should be compared with Fig. 3 for second-order acoustics. Evidently the third-order nonlinearities significantly affect the influences of the linear parameters on stability; Figs. 11 and 12 illustrate two instances: in the first (Fig. 11) the third-order terms are destabilizing, and in the second (Fig. 12) they are stabilizing. However, we must also note that, as often occurs, the amplitudes predicted lie outside the range for which the analysis is physically valid. Such results must be regarded only as suggestions of possible behavior. Clearly the theory is deficient, as one must expect in view of its approximate nature. Based on experience with a limited number of examples, it seems that the amplitudes are usually reduced if more modes are accounted for, although there seems to be an optimum number of modes beyond which the amplitudes are little affected.

In the context of these calculations, triggering to a stable limit cycle means that the trivial stable limit cycle $r_{10}=r_{20}=0$ must be unstable if the initial disturbance is large enough, and the subsequent motion must evolve to a stable nontrivial limit cycle $\left(r_{10}, r_{20}\right.$ $\neq 0$ ). Already we see that this sequence of events is excluded, because the condition $\alpha_{1} \alpha_{2}<0$ for existence of limit cycles forbids the existence of a limit cycle (stable or unstable) for a linearly stable system. Hence accounting for third-order acoustic nonlinearities is not sufficient for the existence of triggering in the sense defined here. We have discussed previously the fact that triggering can be obtained by including nonlinear combustion processes, and so the third-order contributions are certainly not necessary. Thus we conclude that third-order acoustic nonlinearities are neither necessary nor sufficient for the existence of triggering to stable limit cycles.

The conclusion is perhaps most clearly shown by the balance of energy. Following the definitions and procedures introduced in Sec. V.A we find the equations for the rates of change of timeaveraged energies in the lowest two modes:

$$
\begin{aligned}
& \frac{\mathrm{d}\left\langle\varepsilon_{1}\right\rangle}{\mathrm{d} t}=\alpha_{1} \omega_{1}^{2} r_{1}^{2}-\beta \omega_{1}^{2} r_{1}^{2} r_{2} \cos \Psi \\
& \frac{\mathrm{d}\left\langle\varepsilon_{2}\right\rangle}{\mathrm{d} t}=\alpha_{2} \omega_{2}^{2} r_{2}^{2}+\beta \omega_{2}^{2} r_{1}^{2} r_{2} \cos \Psi
\end{aligned}
$$

Hence again the total coupling terms do not affect the total energy $\left\langle\mathcal{E}_{1}\right\rangle+\left\langle\mathcal{E}_{2}\right\rangle$ in the system. Upon substitution of Eqs. (91a) 


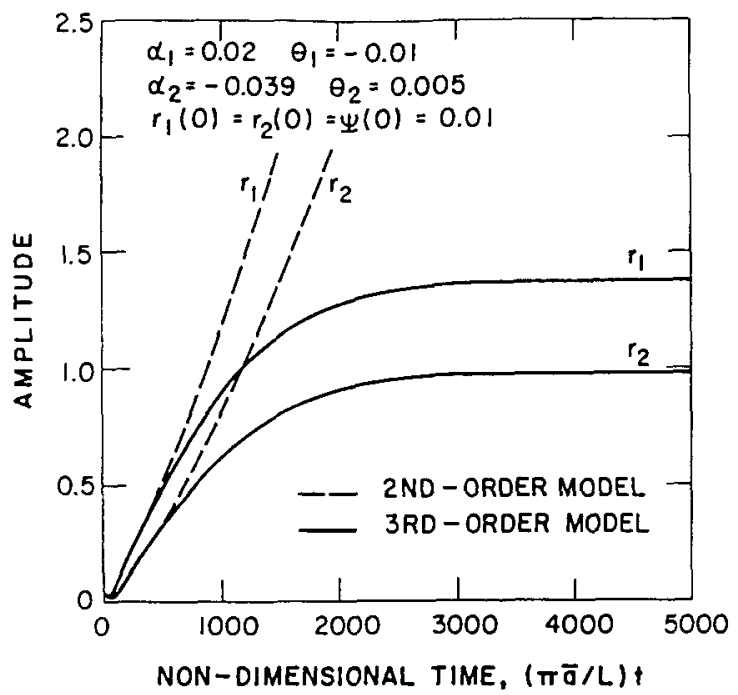

Fig. 12 Example showing stabilizing effects of third-order acoustics: two longitudinal modes, time-averaged equations.

and (91b) in Eqs. (92a) and (92b) we find that the total energy is constant in stable limit cycles.

\section{Transverse Modes}

Similar results are found for transverse modes. ${ }^{39,40}$ As for longitudinal modes, third-order acoustic coupling affects the ranges of linear parameters for which stable limit cycles exist but do not provide the opportunity for triggering to stable limit cycles. The details of the analysis become complicated, and there is nothing to be gained with their inclusion here. Figure 13 shows the influence of the third-order terms on a stable limit cycle of the 1T/1R system (cf. Fig. 6) and Fig. 14 shows the result for a limit cycle involving the $1 \mathrm{~T} / 2 \mathrm{~T}$ pair.

These cases represent much of the behavior for stable limit cycles, but they should not be taken as general in any sense. A significant flaw is that the amplitudes found with the two-mode approximation are often unacceptably large, the actual values depending, of course, on the values of the linear parameters $\left(\alpha_{n}\right.$, $\theta_{n}$ ). It appears that the large amplitudes are a consequence of truncating the modal expansion, as suggested by the example considered in Sec. VI.A.3 for the 1T/1R/2T system, but this conjecture has not been confirmed.

\section{B. Higher Order Mean Flow/Acoustics Interactions}

$\mathrm{Kim}^{39}$ has shown that if terms linear in the mean flow speed and second order in fluctuations are retained, then the force $F_{n}, \mathrm{Eq}$. (84), contains the additional double series,

$$
F_{n}=\cdots-\sum_{i=1}^{\infty} \sum_{j=1}^{\infty} G_{n i j} \dot{\eta}_{i} \dot{\eta}_{j}
$$

where

$$
G_{n i j}=\frac{1}{\gamma E_{i}^{2} E_{j}^{2}} \int \psi_{i} \nabla\left(\bar{u} \cdot \nabla \psi_{j}\right) \cdot \nabla \psi_{n} \mathrm{~d} V
$$

Interactions between the unsteady and steady flows are often fundamentally important in acoustics; sirens and musical wind instruments are familiar examples. That is true as well in combustion instabilities and for the same reason, namely, that the steady flowfield is a source of energy. It is true that this source is considerably less strong than combustion processes, but the efficiency of coupling may under some circumstances compensate the difference.

So far as the present subject is concerned, we are concerned with the relative importance of third-order acoustical contributions and the mean flow/acoustics interactions. In the case of triggering to stable limit cycles, one might expect the latter to win, apparently because they represent a genuine source of energy, whereas the third-order acoustics, as we have seen, act primarily to transfer energy among the possible modes of oscillation.

If we ignore the terms of third-order acoustics, the equations for two modes are 35

$$
\frac{\mathrm{d} A_{1}}{\mathrm{~d} t}=\alpha_{1} A_{1}+\theta_{1} B_{1}-\beta\left(A_{1} A_{2}+B_{1} B_{2}\right)+7 \beta_{m}\left(A_{1} B_{2}-A_{2} B_{1}\right)
$$

$$
\frac{\mathrm{d} B_{1}}{\mathrm{~d} t}=-\theta_{1} A_{1}+\alpha_{1} B_{1}-\beta\left(A_{1} B_{2}-A_{2} B_{1}\right)-7 \beta_{m}\left(A_{1} A_{2}+B_{1} B_{2}\right)
$$$$
\frac{\mathrm{d} A_{2}}{\mathrm{~d} t}=\alpha_{2} A_{2}+\theta_{2} B_{2}+\beta\left(A_{1}^{2}-B_{1}^{2}\right)+2 \beta_{m} A_{1} B_{1}
$$

$$
\frac{\mathrm{d} B_{2}}{\mathrm{~d} t}=-\theta_{2} A_{2}+\alpha_{2} B_{2}+2 \beta A_{1} B_{1}-\beta_{m}\left(A_{1}^{2}+B_{1}^{2}\right)
$$

where

$$
\beta_{m}=\frac{1}{32} \frac{\bar{u}_{\ell}}{\gamma L}
$$

This value of $\beta_{m}$ is calculated for a flow velocity in the axial direction, increasing linearly from zero to $\bar{u}_{\ell}$ at the exit, length $L$ from the origin.
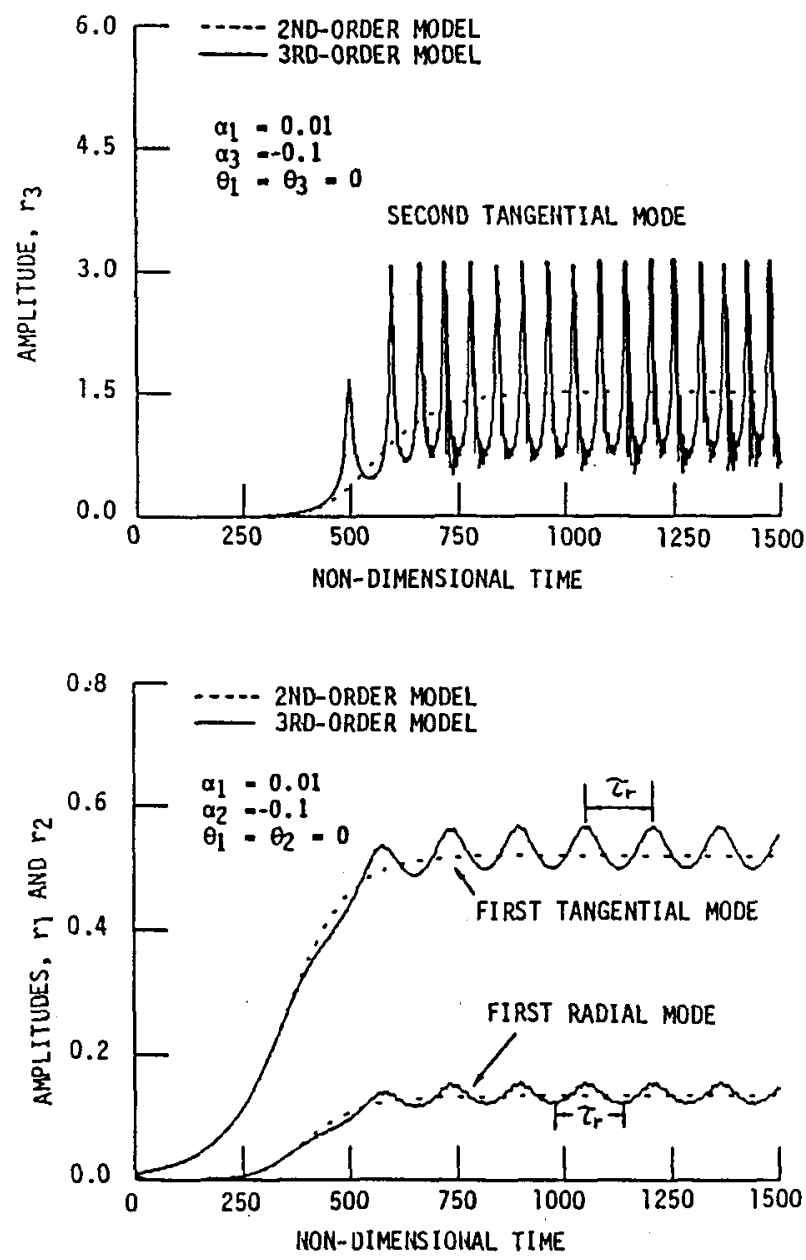

Fig. 13 Example showing some effects of third-order acoustics on a stable limit cycle for the first tangential and first radial modes, timeaveraged equations. 

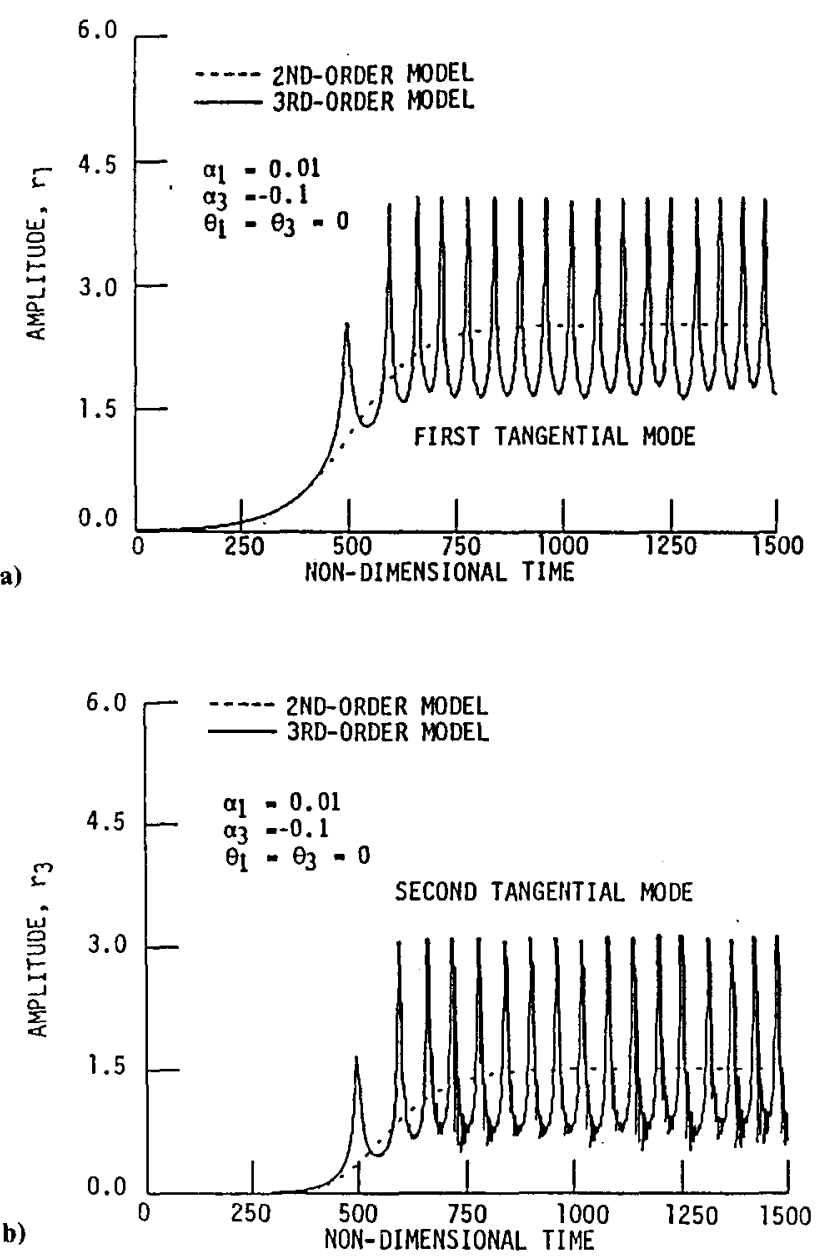

Fig. 14 Example showing some effects of third-order acoustics on a stable limit cycle for the first and second tangential modes, time-averaged equations.

Now the equations for the amplitudes and combined phase angles are

$$
\begin{gathered}
\frac{\mathrm{d} r_{1}}{\mathrm{~d} t}=\alpha_{1} r_{1}-r_{1} r_{2}\left(\beta \cos \Psi+7 \beta_{m} \sin \Psi\right) \\
\frac{\mathrm{d} r_{2}}{\mathrm{~d} t}=\alpha_{2} r_{2}+r_{1}^{2}\left(\beta \cos \psi+\beta_{m} \sin \Psi\right) \\
\frac{\mathrm{d} \Psi}{\mathrm{d} t}=2 \theta_{1}+\theta_{2}+\beta\left(2 r_{2}-\frac{r_{1}^{2}}{r_{2}}\right) \sin \Psi+\beta_{m}\left(-14 r_{2}+\frac{r_{1}^{2}}{r_{2}}\right) \cos \Psi
\end{gathered}
$$

Once again these equations can be solved to give $r_{10}, r_{20}$, and $\Psi$ in limit cycles

$$
\begin{gathered}
r_{10}=\left[\frac{-\alpha_{1} \alpha_{2}}{\left(\beta \cos \Psi_{0}+7 \beta_{m} \sin \Psi_{0}\right)\left(\beta \cos \Psi_{0}+\beta_{m} \sin \Psi_{0}\right)}\right]^{1 / 2} \\
r_{20}=\frac{\alpha_{1}}{\beta \cos \Psi_{0}+7 \beta_{m} \sin \Psi_{0}} \\
\Psi_{0}=\tan ^{-1}\left[\frac{-a_{3} \pm \sqrt{a_{3}^{2}-4 a_{1} a_{2}}}{2 a_{2}}\right]
\end{gathered}
$$

where now

$$
\begin{gathered}
a_{1}=\left(-2 \theta_{1}+\theta_{2}\right) \beta^{2}-\left(14 \alpha_{1}+\alpha_{2}\right) \beta \beta_{m} \\
a_{2}=7\left(-2 \theta_{1}+\theta_{2}\right) \beta_{m}^{2}+\left(2 \alpha_{1}+\alpha_{2}\right) \beta \beta_{m} \\
a_{3}=8\left(-2 \theta_{1}+\theta_{2}\right) \beta \beta_{m}+\left(2 \alpha_{1}+\alpha_{2}\right)\left(\beta^{2}-7 \beta_{m}^{2}\right)
\end{gathered}
$$

The requirements that $r_{10}$ and $\Psi_{0}$ be real provide necessary and sufficient conditions for the existence of limit cycles: the arguments of the square roots must be positive. When $\alpha_{1}, \alpha_{2}<0$, one mode is stable and the other is unstable, we have a situation like those treated earlier, growth of small disturbances into stable or unstable limit cycles. The details are unimportant here (see Yang et al. $^{35}$ and $\mathrm{Kim}^{39}$ ). As before, we find conditions under which the limit cycles are stable, illustrated in Fig. 15, to be compared with Fig. 3 for second-order acoustics and Fig. 10 for thirdorder acoustics. The three cases do not differ in truly significant respects.

Moreover, it is still not possible to find triggering to a stable limit cycle. The limit cycles are still unique, with the difference that there are now limits such that if the initial disturbance has amplitude below those limits the limit cycle is unstable. More precisely, for given values of the linear parameters, when one mode is stable and the other unstable, a unique limit cycle exists, characterized by the two amplitudes $r_{10}^{s}$ and $r_{20}^{s}$. If the initial disturbance is such that both amplitudes $r_{1}, r_{2}$ are below the smaller of $r_{10}^{s}$ and $r_{20}^{s}$, then the final motion is a stable limit cycle. But if both initial amplitudes are greater than the larger of $r_{10}^{s}$ and $r_{20}^{s}$, then the final motion is unstable. Intermediate cases may be stable or unstable, depending on the values of the amplitudes and the relative phases.
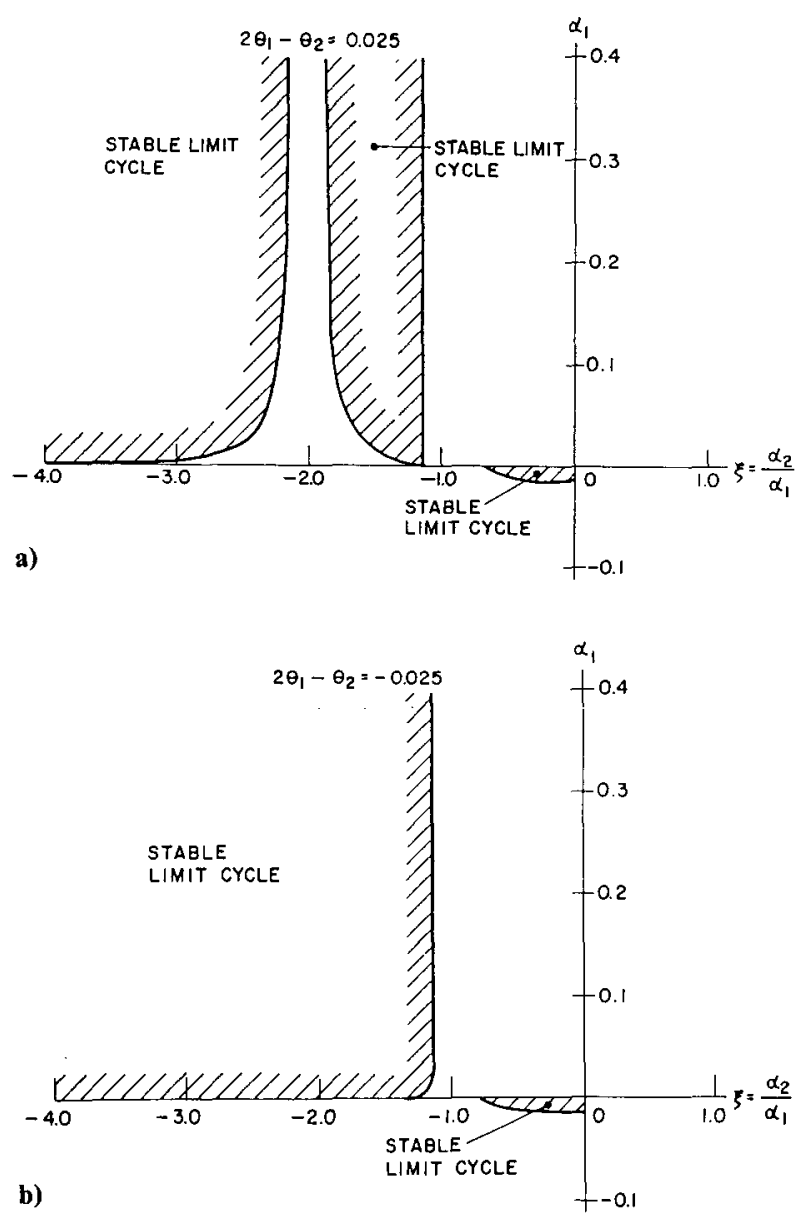

Fig. 15 Regions for stable limit cycles: two longitudinal modes with mean flow/acoustics interactions, time-averaged equations. 
This is an interesting result consistent with some of the numerical results reported by Levine and Baum, ${ }^{37}$ showing dependence of nonlinear behavior on the harmonic context of the initial disturbance. It is not possible at this time either to make a firm correspondence between the two sets of results or to offer any generalizations.

Perhaps the most important conclusion of these calculations is that the mean flow/acoustics interactions, to the order considered here, apparently do not contain triggering to stable limit cycles. As for the result with third-order acoustics, we have found ranges of the linear parameters for which a linearly stable system can be pulsed to unstable limit cycles. Following a somewhat different approach, Paparizos and Culick ${ }^{43}$ have independently reached the same conclusion.

\section{Two-Mode Approximation}

The approximate analysis is intended to serve two main purposes: to provide an efficient means of performing routine calculations for analysis and design and to furnish a theoretical framework within which observed behavior of combustion instabilities may be understood. Even with the formal representation reduced to a set of coupled first-order equations, it is difficult to extract the sort of qualitative information necessary to satisfactory understanding. Accordingly, much effort has been expended in the past few years on the simplest possible case, the approximate model in which only two modes are accounted for. Most of the discussion in the preceding sections of this paper has been based on that model, treated essentially with the method first executed by Awad. ${ }^{36}$ It is remarkable, a consequence of the special form of the gasdynamical nonlinearities, that simple explicit results can be obtained for a useful variety of special problems.

\section{A. Dynamical Systems Theory and Two-Mode Approximation}

In this section we discuss briefly important results obtained recently by Paparizos and Culick. ${ }^{43,44}$ The conclusions are identical or consistent with those cited earlier for the same problems, but the context of the analysis is different, lying wholly within the contemporary geometrical theory of nonlinear dynamical systems. This provides a different view of the nonlinear acoustics and suggests convenient ways for determining the influences of, say, higher order acoustics and mean flow/acoustics interactions. Moreover, the formulation lends itself in a natural way to application of numerical methods for analyzing systems having many degrees of freedom. ${ }^{45}$

The case of two modes is especially notable because the system is described by four first-order equations which, owing to an arbitrary phase, can be reduced to three. In general, for $N$ modes, the $2 N$ equations can be reduced to $2 N-1$, but when there are only three equations, the solutions can be represented completely in three-dimensional diagrams. Graphical presentation of results becomes troublesome for three or more degrees of freedom, but a deeper concern is that fundamental differences arise in the theory (not discussed here) and, therefore, presumably in the physical behavior. How significant those complications may be for acoustical systems is a matter of current study. However, one must be aware that results obtained for two degrees of freedom (two modes) may not accurately represent the behavior for actual systems having many degrees of freedom.

Paparizos and Culick ${ }^{43}$ have shown that the four equations for the two-mode approximation and second-order acoustics can be written as the reduced set of three equations

$$
\begin{gathered}
\dot{y}_{1}=\left(\alpha_{1}+y_{2}\right) y_{1} \\
\dot{y}_{2}=\alpha_{2} y_{2}+\left|\theta_{2}-2 \theta_{1}\right| y_{3}+w y_{3}^{2}-y_{1}^{2} \\
\dot{y}_{3}=-\left|\theta_{2}-2 \theta_{1}\right| y_{2}+\alpha_{2} y_{3}-2 y_{2} y_{3}
\end{gathered}
$$

with the transformation of variables from amplitudes and phases to $\left(y_{1}, y_{2}, y_{3}\right)$

$$
\begin{gathered}
y_{1}=\beta r_{1} \\
y_{2}=\beta r_{2} \sin \left(\phi_{2}-2 \phi_{1}\right) \\
y_{3}=\beta \frac{\left|\theta_{2}-2 \theta_{1}\right|}{\theta_{2}-2 \theta_{1}} r_{2} \cos \left(\phi_{2}-2 \theta_{1}\right)
\end{gathered}
$$

In these variables, the amplitudes in the limit cycles are

$$
\begin{gathered}
y_{10}=\sqrt{-\alpha_{1} \alpha_{2}}\left[1+\left(\frac{\theta_{2}-2 \theta_{1}}{\alpha_{2}+2 \alpha_{1}}\right)^{2}\right]^{1 / 2} \\
y_{20}=-\alpha_{1} \\
y_{30}=-\frac{\alpha_{1}\left(\theta_{2}-2 \theta_{1}\right)}{\alpha_{2}+2 \alpha_{1}}
\end{gathered}
$$

The conditions for existence and stability can be established for all values of the linear parameters, providing the basis for the regions of stable limit cycles shown in Fig. 16.

These results are of course identical with those discussed in Sec. $\mathrm{V}$, although the representation is different. In particular, stable limit cycles are unique; their global dynamic character is conveniently displayed in the $\left(y_{1}, y_{2}, y_{3}\right)$ space, Fig. 17 , or drawn for the case when the first mode is unstable and the second is stable. A curve representing a trajectory-i.e., the time evolution of the system subsequent to a chosen initial condition-converges to a state representing a limit cycle lying in the line, or one-dimensional manifold, $O P$. In the context of the modern theory of dynamical systems, $O P$ is a center manifold. A point on the center manifold is uniquely specified by the values of the linear parameters, corresponding to the uniqueness of limit cycles. The origin is a Hopf bifurcation. If both $\alpha_{1}$ and $\alpha_{2}$ are negative, the "limit cycle" is the quiescent state, $y_{i}=0$. But if $\alpha_{1}>0$ or $\alpha_{2}>0$ and the values of all of the parameters satisfy the stability condition, then $y_{1}, y_{2}, y_{3} \neq 0$ and the system executes a limit cycle.

Paparizos and Culick have shown that the center manifold can be closely approximated by interpreting the activity in the limit cycle as the second mode being driven by the first mode. That is a reinterpretation of the characteristic described earlier that these systems are strongly influenced by the tendency for energy to flow from low to high frequencies. Formally the equation for the center manifold-the locus of points representing limit cycles-is obtained by fixing $y_{1}$, and solving Eqs. (105b) and (105c) for $y_{2}$ and $y_{3}$ when $\dot{y}_{2}=\dot{y}_{3}=0$. The two equations can be combined to

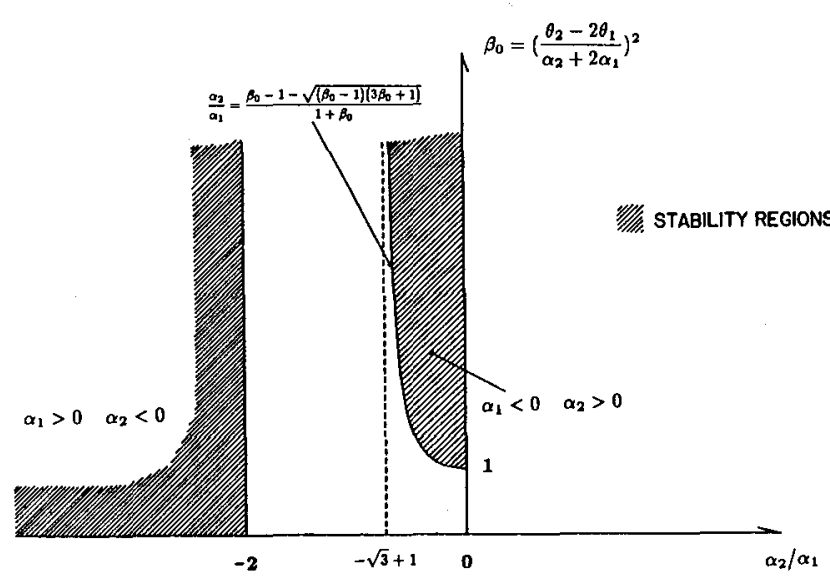

Fig. 16 Regions for stable limit cycles: two longitudinal modes, timeaveraged equations. 
give a third-order polynomial in $y_{2}$. Figure 18 shows the result projected into the $y_{2}-y_{3}$ plane.

Most of the results reported by Paparizos and $\mathrm{Culick}^{43}$ are directed to assessing the limits of the two-mode approximation. It provides good results for the amplitudes in limit cycles if the first mode is unstable (hence the second must be stable) and if the combination of linear parameters does not lie too close to the stability boundary in Fig. 16. Difficulties arise when the second mode is unstable (so the first mode is stable) because the absence of higher modes blocks the natural tendency for upward flow of energy. Thus, although the amplitudes in the limit cycle may not be badly approximated (although it appears that usually their values will be quite inaccurate), the transient development of the motion may be wholly unrealistic. Fig. 19 shows an example.

The consequences of truncation are illustrated explicitly with Figs. 20 and 21 for the two cases $\left(\alpha_{1}>0, \alpha_{2}<0\right)$ and $\left(\alpha_{1}<0, \alpha_{2}\right.$ $>0$ ). Obviously the two-mode approximation is good in the first instance and simply does not work for this example of an unstable second mode. In the second case, the limit cycle is, of course, predicted to be unstable by the conditions derived earlier, but the point is that including higher modes leads to a stable limit cycle. That is, as one must anticipate, the conditions for existence and stability of limit cycles depends on the number of modes accounted for.

\section{B. Application of Bifurcation Theory and Continuation Method}

Practically all problems arising with application of the approximate analysis involve determining the behavior of unsteady motions in a combustor as the values of parameters are changed. The simplest example is linear stability: the system becomes linearly unstable if one or more decay constants become positive. Much of the preceding discussion thus dealt with aspects of nonlinear behavior as various parameters were varied.

It is an immense task to discover all possible sorts of behavior due to the large number of parameters: for a given geometry, there are two for each mode considered plus whatever quantities are

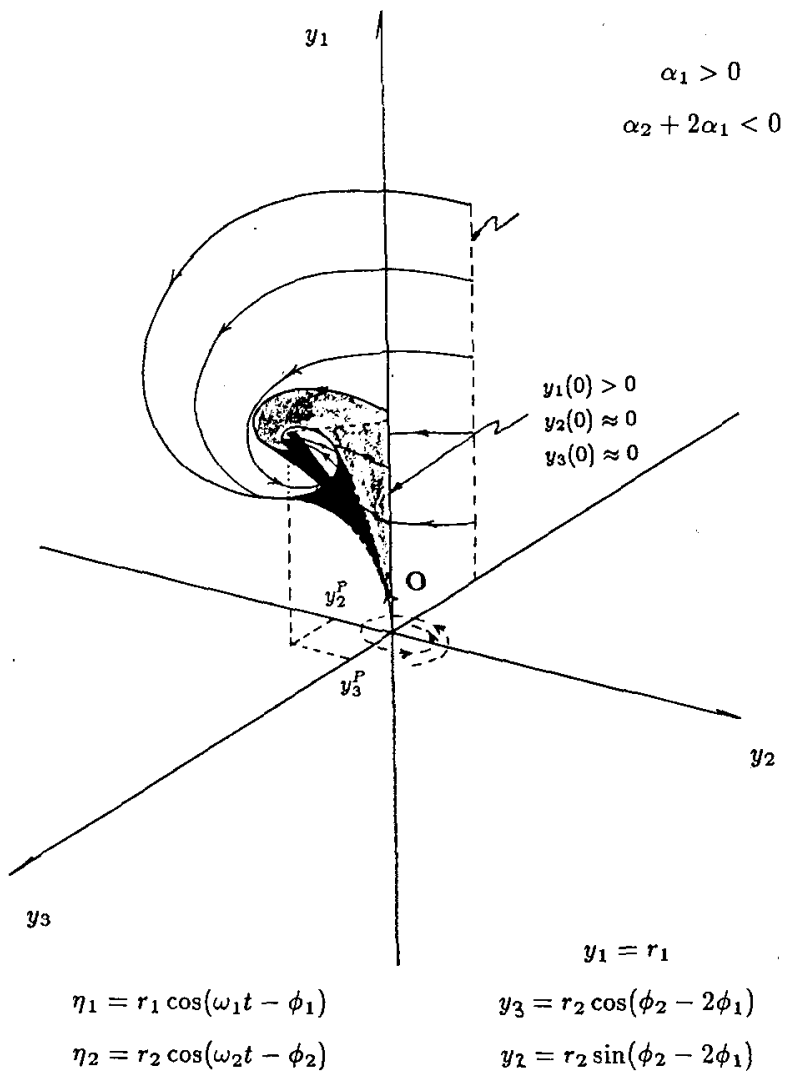

Fig. 17 Trajectories for the development of a stable limit cycle on the center manifold.

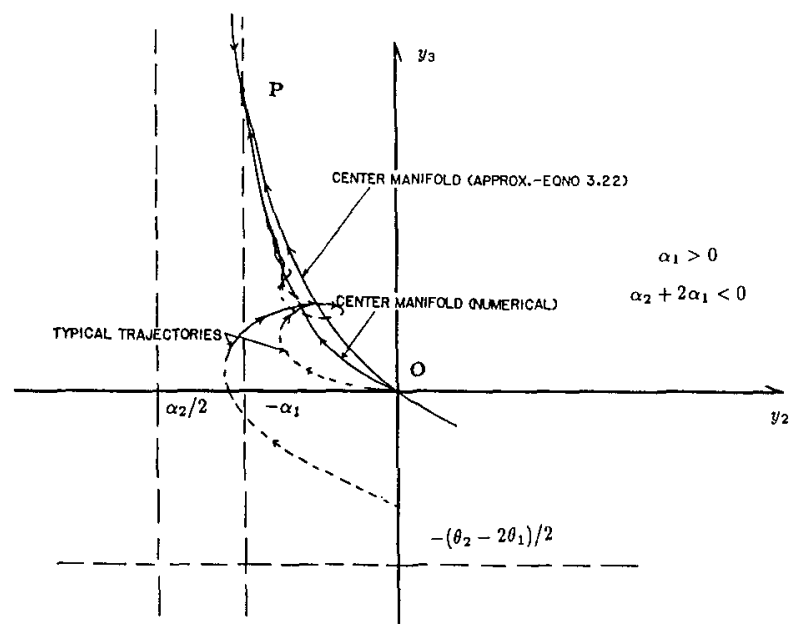

Fig. 18 Picture of the center manifold approximation.

introduced by nonlinear processes. (Note that nonlinear gas dynamics alone does not bring any new parameters, in addition to those set by the geometry, if the mean flow is not accounted for.) That is why exact solutions, such as those for the two-mode approximation, are so important. However, only a few exact solutions have been found, and only for problems restricted in three important aspects: the only nonlinear process is gasdynamics; the modal expansion is truncated to a small number of modes; and the time-averaged equations have been used. For both theoretical purposes and for practical applications, it is essential to avoid these restrictions.

In the absence of analytical solutions, it is necessary to resort to numerical methods. Numerical simulations for the system (30) of coupled oscillators can be carried out without serious difficulties, but that is an expensive procedure. It is more efficient and productive of useful information to apply a continuation method for computing stationary states (e.g., limit cycles) of the dynamical system as one or more parameters are changed. Well known in other fields, the approach has been adapted by Jahnke and Culick ${ }^{45}$ to investigate nonlinear combustion instabilities. The procedure is given broadly in the following, for the case of longitudinal modes and only nonlinear second-order acoustics.

First the system (30) is put in the form

$$
\dot{x}=f[x, \mu(\varepsilon)]
$$

where $x$ is a vector of dependent variables (the state vector); $\mu$ represents the parameters $\left(\alpha_{n}, \theta_{n}\right)$; and $\varepsilon$ is a small parameter, here of the order of the average Mach numbers. After rescaling time, $t$ $\rightarrow \omega_{1} t$ and defining the variable $\delta_{n}=\dot{\eta}_{n}$, the Eqs. (30) with $F_{n}$ written for longitudinal modes, can be put in the form (108), with $x$ $=\left(\eta_{n}, \delta_{n}\right)$

$$
\begin{aligned}
\dot{\eta}_{n} & =\delta_{n} \\
\dot{\delta}_{n} & =-n^{2} \eta_{n}+2 \alpha_{n} \delta_{n}+2 n \theta_{n} \eta_{n} \\
& =-\sum_{i=1}^{n-1}\left[C_{n i}^{(1)} \delta_{i} \delta_{n-1}+D_{n i}^{(1)} \eta_{i} \eta_{n-i}\right] \\
& =-2 \sum_{i=1}^{\infty}\left[C_{n i}^{(2)} \delta_{i} \delta_{n-1}+D_{n i}^{(2)} \eta_{i} \eta_{n+i}\right]
\end{aligned}
$$

To obtain numerical results, the modal expansion must be truncated at some number of modes $n=N$. Following the method outlined in Sec. II.B, or similar procedures, the system (109) can also be time averaged. By carrying out calculations for both Eqs. (109) and the time-averaged system, and for increasing $N \geq 2$, it is then 


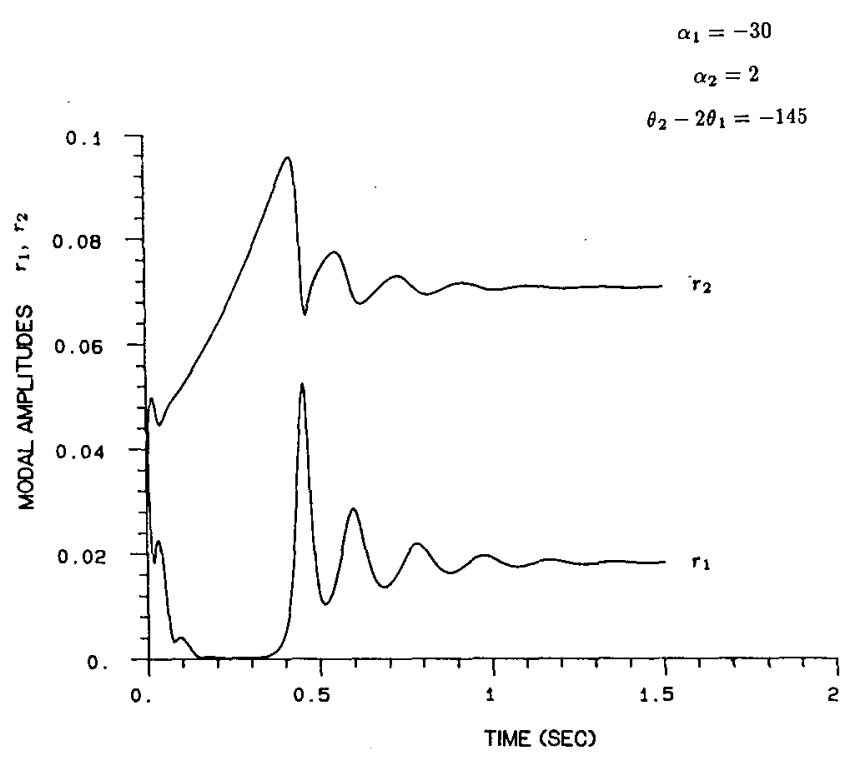

Fig. 19 Example of evolution to a limit cycle: unstable second mode.

possible to determine the consequences of modal truncation and time averaging.

The next step is to determine the stationary states defined by $\dot{x}$ $=0$, the solution to

$$
f(x, \mu)=0
$$

or the corresponding time-averaged equation. This is of course exactly what was done in Secs. V-VII to determine the existence of limit cycles. In general, solving Eq. (110), which is a set of $N$ coupled nonlinear equations, becomes difficult. A continuation method (we have used that worked out by Doedel and Kernevez ${ }^{46}$ ) is a recipe for finding the values of the dependent variables $x_{0}$ defining the stationary states as the parameters $\mu$ are varied ("continued") from some initial values. At each stage, after the values of $\mu$ have been changed, the stability of the new stationary state is determined by computing the eigenvalues of the Jacobian matrix arising in the linearized problem. That process also identifies bifurcations.

Use of a continuation method does not resolve the difficulty of determining the behavior over broad ranges of many parameters. However, the procedure does impose a certain systematic approach and yields much more information than one obtains from numerical simulations alone. One example makes the point.

The two-mode approximation with time averaging produces the result summarized in Fig. 16: stable limit cycles exist only if the values of the parameters $\alpha_{n}, \theta_{n}(n=1,2)$ lie in certain ranges defined by a stability boundary. It is not possible within that restricted analysis to state whether the presence of the stability boundary is intrinsic to the physical mode, or is due to the approximations of modal truncation and/or time averaging. With the continuation method we have partly resolved the question. For a particular set of fixed parameters $\left(\theta_{1}, \alpha_{2}, \theta_{2}\right)$ and $\alpha_{1}$ varied, the stability region for the original equations without time averaging is reduced and, in fact, a turning point bifurcation appears as $\alpha_{1}$ is increased from zero.

More interestingly and significant is the result, again for fixed values of the other parameters, that the stability boundary apparently does not exist if enough modes are accounted for. Figures 20 and 21 already suggest the anticipated importance of truncation. With the continuation method, the effect of truncation can be found in a straightforward fashion. The values of all of the parameters except $\alpha_{1}$ are fixed $\left(\alpha_{n}<0\right.$ for $\left.n \geq 2\right)$ and $\alpha_{1}$ is varied from zero so that the first mode is linearly unstable. Figs. 22 and 23 show the amplitudes for the cases of two modes and four modes. With the averaged equations and the two-mode approximation, the amplitudes become infinite when $\alpha_{1}=131$ (i.e., on the stability boundary). However, the second-order equations have a turningpoint bifurcation at $\alpha_{1}=131$, Fig. 22 .

In contrast, when four modes are accounted for, as shown in Fig. 23, there is neither a stability boundary nor a bifurcation for $\alpha_{1}$ less than 300. No results have been computed in this case for the time-averaged equations although other examples suggest that the region of stability is at least expanded when more modes are included.

Further details and examples are given by Jahnke and Culick. ${ }^{45}$ It seems at this time that the application of a continuation method offers the best means of understanding the global behavior of the dynamical system (30) representing combustion instabilities. Work is in progress to include other nonlinear processes and to obtain results for modes other than purely longitudinal.

\section{Influences of Third-Order Acoustics, Mean Pressure Shifts, and} Mean Flow/Acoustic Interactions in the Two-Mode Approximation

The viewpoint developed by Paparizos and discussed at length by Paparizos and Culick ${ }^{43,44}$ provides a convenient means of investigating nonlinear perturbations beginning with the basic problem of second-order acoustics. A particular advantage is the geometrical interpretation and, for the two-mode approximation, the opportunity to display trajectories in the three-dimensional $\left(y_{1}, y_{2}, y_{3}\right)$ space. The ability to view the global dynamics, as in the analysis of Jahnke and Culick, brings with it much increased understanding of the behavior of these systems.

In addition to the center manifold shown in Fig. 18, there is a second attractive manifold. The two manifolds are determined by considering the case of an unstable fundamental mode and stable second mode. Then, as noted earlier, the equation for the amplitude of the second mode can be interpreted as that for a damped oscillator driven by the nonlinear transfer of energy from the fundamental mode. The equations for the attractive manifolds are then given approximately by considering $y_{1}$ parameter and setting $\dot{y}_{2}=$ $\dot{y}_{3}=0$ in Eqs. (82b) and (82c); then $y_{2}\left(y_{1}\right)$ and $y_{3}\left(y_{1}\right)$ are the solutions to the algebraic equations:

$$
\begin{aligned}
& \alpha_{2} y_{2}+\left|\theta_{2}=2 \theta_{1}\right| y_{3}+2 y_{3}^{2}-y_{1}^{2}=0 \\
& -\left|\theta_{2}-2 \theta_{1}\right| y_{2}+\alpha_{2} y_{3}-2 y_{2} y_{3}=0
\end{aligned}
$$

Figure 23 shows a typical result for the two manifolds, drawn in the $\left(y_{1}, y_{2}, y_{3}\right)$ space and projected in the $y_{2}-y_{3}$ plane.

The existence of the second attractive manifold is a new result, crucial to understanding the global nonlinear behavior of this system. Within this framework, three important influences have been investigated: third-order acoustics, shifts of the mean pressure, and nonlinear mean flow/acoustics interactions. The results generally agree with those discussed earlier for third-order acoustics and mean flow/acoustics interactions, but the geometrical representation offers much additional insight. The question of possible con-

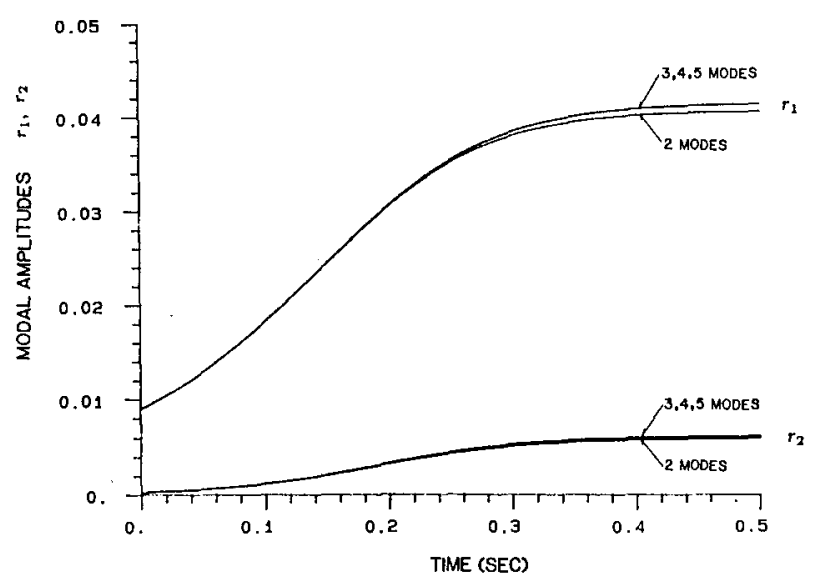

Fig. 20 Example showing small effects of truncation. 
sequences of accommodating shifts of the mean pressure has barely been touched upon. It is a highly important matter for solid propellant rockets, since dc shifts are often observed, they are possibly (if not likely) to be indicative of some sort of effect due to velocity coupling and often seem to be connected with triggering to stable limit cycles. This is a problem that has considerable practical implications and may as well raise interesting theoretical questions.

\section{Rayleigh's Criterion}

In 1878 Lord Rayleigh ${ }^{47}$ formulated his famous criterion to explain several examples of acoustic waves excited and maintained by heat addition:

If heat be communicated to, and abstracted from, a mass of air vibrating (for example) in a cylinder bounded by a piston, the effect produced will depend upon the phase of the vibration at which the transfer of heat takes place. If heat be given to the air at the moment of greatest condensation, or be taken from it at the moment of greatest rarefaction, the vibration is encouraged. On the other hand, if heat be given at the moment of greatest rarefaction, or abstracted at the moment of greatest condensation, the vibration is discouraged.

Probably no principle in the subject of thermally excited acoustic waves has been more frequently called upon than this concise statement. The physical basis of the principle, and hence its interpretation, is the following.

Consider a small volume element within a gas sustaining unsteady motions. An amount of heat added to the volume causes the temperature to rise and the density to fall, i.e., the volume element expands. If at the same time the surrounding atmosphere is expanding (i.e., the pressure and density are falling), then the material that has gained the heat will do work on the surrounding medium as it expands. Thus some portion of the energy associated with the heat addition is converted to mechanical energy of motion in the medium. In this way, heat addition can drive and sustain acoustic waves. A steady source of heat will not generate acoustic waves: in the wave equation (21), the term on the right-hand side representing the effect of heat addition is $-\left(\bar{R} / \bar{C}_{v} \bar{a}^{2}\right)\left(\partial Q^{\prime} / \partial t\right)$ where $Q^{\prime}$ is the fluctuation of heat added per unit volume and time.

Most of the applications of Rayleigh's criterion have been based on linear behavior (see Putnam ${ }^{48,49}$ for the most extensive discussions), and usually the results have been obtained in a somewhat heuristic fashion. The only careful derivations in the context of stability theory seem to be those due to $\mathrm{Chu}^{18}$ and $\mathrm{Zinn}^{50}$ who provided a linear version of the derivation later worked out by Culick $^{51}$ for nonlinear behavior.

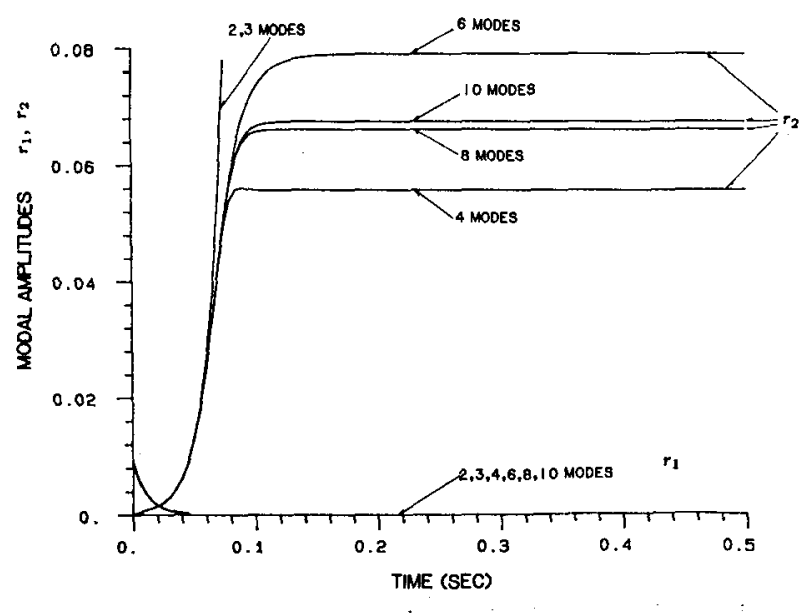

Fig. 21 Example showing drastic effects of truncation.
Two Mode Approximation

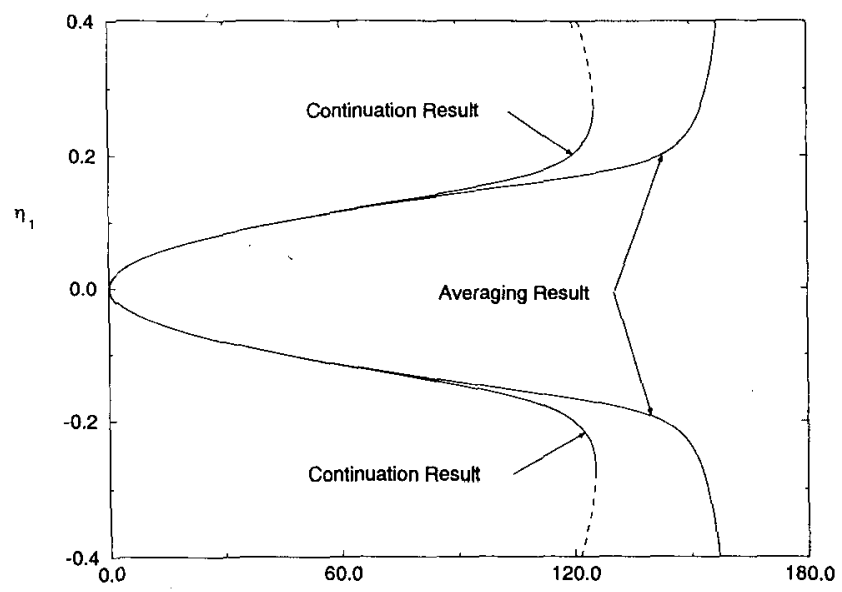

a)

$\alpha$,

Two Mode Approximation

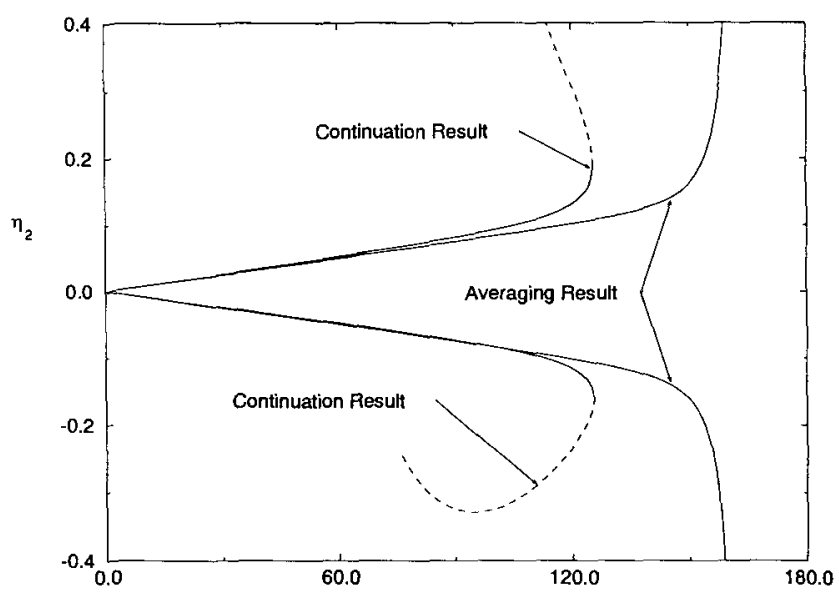

b)

Fig. 22 Comparison of results from the time-averaged equations and from the continuation method.

Before examining the matter for nonlinear motions, we note a basic connection between Rayleigh's criterion and the growth constant. ${ }^{6,52}$ The criterion phrased in terms of heat addition is really only a special case; because Rayleigh was specifically concerned with the action of heat addition, he paid no attention to other possible gains and losses of energy. Historical emphasis on Rayleigh's statement-perhaps partly because it was Rayleigh who wrote the words-has obscured the fact that the criterion in the form given earlier is exactly equivalent to the contribution of heat addition to the growth constant computed in stability theory, expressed here as Eq. (41b). It follows that the criterion can be expressed in a form accommodating all processes affecting stability. To see this, we begin with Eq. (30) for the time-dependent amplitude of the $\mathrm{n} t h$ mode, $\ddot{\eta}_{n}+\omega_{n}^{2}=F_{n}$. According to the reasoning in Sec. V.A, we take $\varepsilon_{n}=\left(\dot{\eta}_{n}^{2}+\omega_{n}^{2} \eta_{n}^{2}\right) / 2$ to be the energy of the oscillator associated with the $\mathrm{n} t h$ mode; within a constant multiplier, $\varepsilon_{n}$ is the acoustic energy for the nth unperturbed mode. As a result of the "force" $F_{n}$, energy flows into the $n$th mode at the rate $F_{n} \dot{\eta}_{n}$; thus, at time $t$, the change in energy in one period $\tau_{n}=2 \pi / \omega_{n}$ of the oscillation is

$$
\Delta \varepsilon_{n}(t)=\int_{t}^{t+\tau_{n}} F_{n} \dot{\eta}_{n} \mathrm{~d} t^{\prime}
$$




\section{Four Mode Approximation}

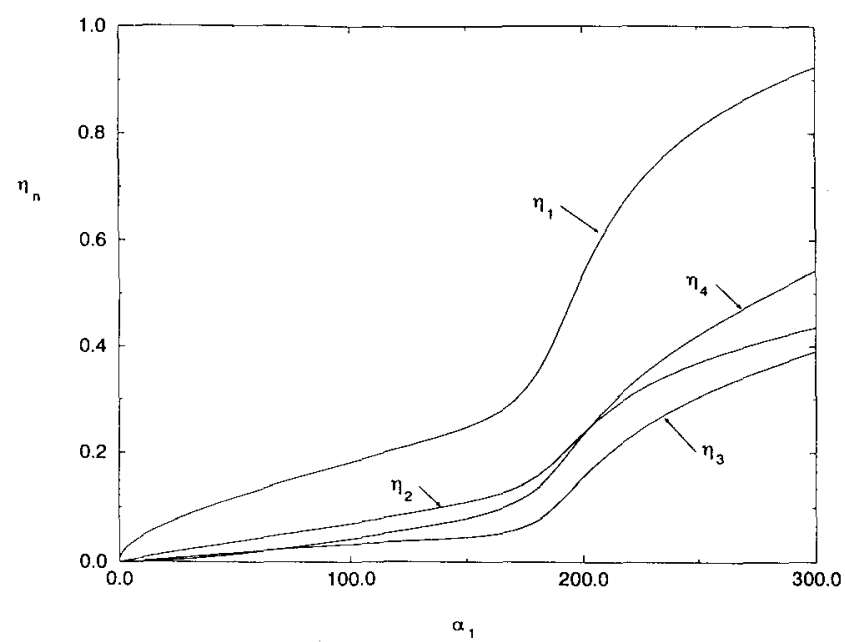

Fig. 23 Example showing the effects of including larger numbers of modes.

Substitution of $\eta_{n}=\hat{\eta}_{n} e^{i \bar{a} k t}$ and $F_{n}=\hat{F}_{n} e^{i \bar{a} k t}$ for linear behavior reduces Eq. (30) to the formula for the complex wave number, $k^{2}$ $=\omega_{n}^{2}-\hat{F}_{n} / \bar{a}^{2}$. The real and imaginary parts are

$$
\begin{aligned}
& \omega^{2}=\omega_{n}^{2}-\hat{F}_{n}^{(r)} \\
& \alpha_{n}=\frac{1}{2 \omega_{n}} \hat{F}_{n}^{(i)}
\end{aligned}
$$

where we have used the fact, as in Sec. IV, that $\alpha_{n} / \omega \ll 1$, and we write here $\alpha_{n}$ as the value of $\alpha$ for the $n$th mode.

Integration of the right-hand side of Eq. (111) by parts and substitution of Eq. (112b) leads eventually to the formula

$$
\Delta \mathcal{E}=2 \pi \omega_{n} \alpha_{n}
$$

Thus if we take $\Delta \tilde{F}_{n}$ to be the measure of energy change implied in Rayleigh's criterion, this equation establishes the equivalence between that principle and the general result of linear stability: if $\alpha_{n}>0$, then $\Delta \varepsilon_{n}>0$ and the mode is unstable. The result applies to any small disturbance synthesized from its modal components.

To extract the special case for heat addition, the original form of Rayleigh's criterion, we need only select that part of $F_{n}$ arising from heat addition. From the definitions (31), (22), (12), and (11), we find the term in question to be

$$
F_{n}=\frac{\bar{R} / \bar{C}_{v}}{\tilde{p} E_{n}^{2}} \int \frac{\partial Q^{\prime}}{\partial t} \dot{\eta}_{n} \psi_{n} \mathrm{~d} V
$$

Substitute this term only in Eqs. (111) and integrate by parts in time to give

$$
\Delta E_{n}=\frac{\bar{R} / \bar{C}_{v}}{\bar{p} E_{n}^{2}} \int\left\{\left[Q^{\prime} \dot{\eta}_{n}\right]_{t}^{t+\tau_{n}}-\int_{t}^{t+\tau_{n}} Q \ddot{\eta}_{n} \mathrm{~d} t^{\prime}\right\} \Psi_{n} \mathrm{~d} V
$$

The product $Q^{\prime} \dot{\eta}_{n}$ has (approximately) period $\tau_{n}$ and so the first term vanishes; in the second, we set $\ddot{\eta}_{n} \approx-\omega_{n}^{2} \eta_{n}$, true to the same approximation, and

$$
\Delta \varepsilon_{n}=(\bar{\gamma}-1) \frac{\omega_{n}^{2}}{\bar{p} E_{n}^{2}} \int \mathrm{d} V \int_{t}^{t+\tau_{n}} Q^{\prime}\left(t^{\prime}\right) \Psi_{n} \mathrm{~d} t^{\prime}
$$

This is the explicit expression of Rayleigh's criterion.

In fact, the formula (115) is not restricted to linear behavior. Although the motions are required to be "small" in the sense implied by the approximations used in formulating the approximate analysis, nothing in the derivation, from the definition (111) to the result (115) prevents consideration of nonlinear behavior. For nonlinear problems, $Q^{\prime}(t)$ cannot usually be expressed as a linear superposition of contributions associated with each of the normal modes. Explicit results can be obtained only by solving the nonlinear problem according to the procedures discussed in the earlier parts of this paper; $\Delta \tilde{E}_{n}$ will then depend, generally, on the amplitude of the $n$th mode.

In the past several years, the formula (115) has been successfully used in interpreting experimental results. Generally, it is assumed that $Q^{\prime}$ is proportional to measurements of the radiation field associated with the combustion processes. It is then possible to confirm that the acoustic oscillations are in fact being driven by the energy addition (see Sterling ${ }^{53}$ and Sterling and Zukoski ${ }^{54}$ for examples of the procedure).

\section{$X$. Nonlinear Acoustics, Chaos, and Noise}

Developments in the theory of nonlinear dynamical systems during the past two decades have encouraged new interpretations of experimental results as well as different methods of analysis. Measured time histories of the pressure in a combustion chamber will always show aspects of randomness as well as contributions from well-defined oscillations. If combustion instabilities are present, the power spectrum will show peaks rising from a broadband background.

The traditional explanation of power spectra of this sort has been based on the assumption that the two important contributions are noise and acoustic oscillations, perturbed by the various processes we have discussed or alluded to in the preceding sections. "Noise" is presumably associated with flow separation, turbulence, random fluctuations of combustion processes, and so forth. However, dynamical systems theory suggests that deterministic chaos may be a third contributor: a deterministic nonlinear system may execute aperiodic apparently random motions in the absence of stochastic sources. This possibility poses interesting theoretical questions, and in addition forces the issue of processing experimental data to discover more precisely what activities are in fact significant when combustion instabilities are found.

At least three questions must be answered: 1) Do pressure records show the presence of low-dimensional attractors associated with nonlinear dynamics capable of producing broadband spectra? 2) What influences do stochastic sources (noise) have on nonlinear combustion instabilities? 3) Will the formulation of nonlinear acoustics discussed here predict chaotic behavior? The first item is concerned solely with processing experimental data. Kantor $^{55}$ first proposed that cycle-to-cycle variations in an internal combustion engine may betray the actions of nonlinear dynamics associated with chemical kinetics. His idea was developed further by Daily ${ }^{56}$ who constructed a more realistic, yet still simple, model of the internal flow and combustion processes. Daily concluded that apparently random variations could indeed be caused by nonlinear dynamics. Whether the behavior he computed is truly "chaotic" in the narrow technical sense remains an open question, but the important point has been made that causes other than random noise due to turbulence may contribute significantly to observed power spectra.

More recently, Keanini et al..$^{57}$ have analyzed pressure records taken for a laboratory dump (rapid expansion) combustor to search for possible influences of deterministic nonlinear processes. Technically what they sought was the existence of a "low-dimensional attractor" which if it is "strange," or has a "fractional dimension" suggests the presence of chaos. They conclude that "Preliminary evidence indicates that a low dimensional strange attractor exists under certain conditions during unstable combustion in a laboratory ramjet."

Sterling ${ }^{58,59}$ is currently studying data taken in a laboratory combustor at California Institue of Technology, with the same intentions. It is a delicate process, apparently quite likely to produce 
misleading conclusions. So far, low-dimensional attractors having integer dimensions have been found, but there is no evidence of strange attractors. For a short summary of the results, see also Culick et al. ${ }^{60}$

Little has been done theoretically with the nonlinear systems described in this paper to seek possible chaotic behavior and no evidence has appeared in numerical calculations. Some calculations have been carried out to investigate the effects of stochastic sources in the two-mode approximation. ${ }^{61,62}$ Only incomplete exploratory results have been obtained. ${ }^{60}$

The preceding three questions listed merit serious attention. Present understanding of the processes in a combustion chamber is insufficient to allow complete and unambiguous interpretation of pressure records. Establishing an accurate assessment of the relative contributions of random sources, deterministic chaos (if it exists in these systems), combustion instabilities, and other possible processes to unsteady motions is a necessary prerequisite to the design of effective active control systems.

\section{Concluding Remarks}

The theory of linear combustion instabilities is well-understood and procedures for computing the stability characteristics of practical systems have been shown by experience to be effective. Accurate results can be obtained for the growth or decay of small disturbances if the processes contributing to the energy gains and losses can be faithfully modeled. In most applications, the most difficult problems arise in determining the dominant source of energy gain, i.e., the mechanisms for the instabilities. Usually those processes are directly associated with the energy release accompanying combustion, but various interactions between the unsteady motions and the average flowfield may be significant. Extended discussions of mechanisms may be found in recent reviews. ${ }^{6-8}$

Linear theory provides results only for the initial growth or decay of small disturbances. An unstable motion in a real system will grow until limited by nonlinear processes. Hence, to learn how to prevent unacceptably large amplitudes or to make effective changes in an existing system, it is essential to understand nonlinear behavior. In this paper we have discussed briefly the most important issues arising with nonlinear acoustics in combustion chambers. Results obtained so far have provided partial understanding of the fundamental phenomena and have aided considerably the interpretation of behavior observed in practical systems. However, the greatest potental benefits lie in development of active control systems, a subject just now in its early stage; success will require continued progress in the theory of nonlinear acoustics.

\section{Acknowledgments}

Most of the work described here, both mine and that of others, has been supported for many years, at variable levels, by the Air Force, Navy, and NASA. My students and I have received much support from the California Institute of Technology and currently by the Office of Naval Research, Grant N00014-89-J-1753. I have personally benefitted greatly from close collaborations in the recent past with Jay Levine of the Air Force Astronautics Laboratory; Vigor Yang and Leonidas Paparizos, Pennsylvania State University and Carnegie-Mellon University, respectively; Craig Jahnke, Clarkson University; and James Sterling, California Institute of Technology.

\section{References}

${ }^{1}$ Harrje, D. J., and Reardon, F. H. (eds.), Liquid Propellant Rocket Instability, NASA SP-194, 1972.

${ }^{2}$ Baum, J. D., Levine, J. N., and Lovine, R. L., "Pulse Triggered Nonlinear Combustion Instability in Solid Rocket Motors," AIAA Journal, Vol. 22, No. 10, 1984, pp. 1413-1419.

${ }^{3}$ Baum, J. D., Lovine, R. L., and Levine, J. N., "Ejecta Pulsing of Subscale Solid Propellant Rocket Motors," AIAA Journal, Vol. 23, No. 3, 1985, pp. $416-423$.

${ }^{4}$ Baum, J. D., Levine, J. N., and Lovine, R. L., "Pulsed Instability in Rocket Motors; A Comparison Between Predictions and Experiments,"
Journal of Propulsion and Power, Vol. 4, No. 4, 1988, pp. 308--316.

${ }^{5}$ Culick, F. E. C., "Nonlinear Behavior of Acoustic Waves in Combustion Chambers, Parts I and II," Acta Astronautica, Vol. 3, 1976, pp. 714-757.

${ }^{6}$ Culick, F. E. C., "Combustion Instabilities in Liquid-Fueled Propulsion Systems," AGARD Conference Proceedings, Paper 1, 450, 1988.

${ }^{7}$ Culick, F. E. C., "Combustion Instabilities in Propulsion Systems," ASME Winter Meeting, San Francisco, Oct. 1989.

${ }^{8}$ Culick, F. E. C., and Yang, V., "Prediction of the Stability of Unsteady Motions in Solid Propellant Rocket Motors," Nonsteady Burning and Combustion Stability of Solid Propellants, Progress in Astronautics and Aeronautics, edited by L. de Luca, E. W. Price, and M. Summerfield, AIAA, Washington, DC, 1992, Chap. 15.

${ }^{9}$ Marble, F. E., "Dynamics of Gas Containing Small Solid Particles," Combustion and Propulsion, 5th AGARD Colloquium, Pergamon, New York, 1963, pp. 175-213.

${ }^{10}$ Fung, Y.-T., Yang, V., and Sinha, A., "Active Control of Combustion Instabilities with Distributed Actuators," Combustion Science and Technology, Vol. 78, No. 6, 1991, pp. 217-245.

${ }^{11}$ Chu, B.-T., and Kovasznay, L. S. G., "Nonlinear Interactions in a Viscous Heat-Conducting Compressible Gas," Journal of Fluid Mechanics, Vol. 3, No. 5, 1957, pp. 494-512.

${ }^{12}$ Zinn, B. T., and Powell, E. A., "Application of the Galerkin Method in the Solution of Combustion Instability Problems," Proceedings of the 19th International Astronautical Congress, Vol. 3, 1970, pp. 59-73.

${ }^{13}$ Zinn, B. T., and Powell, F. A., "Nonlinear Instability in Liquid-Propellant Rocket Engines," Thirteenth Symposium (International) on Combustion, Combustion Inst., Pittsburgh, PA, 1971, pp. 491-503.

${ }^{14}$ Yang, V., Lee, W.-T., and Culick, F. E. C., "On the Applicability of the Method of Averaging in Analyses of Pressure Oscillations in Combustion Chambers," unpublished numerical examples, April 1986.

${ }^{15}$ Culick, F. E. C., "Nonlinear Growth and Limiting Amplitude of Acoustic Oscillators in Combustion Chambers," Combustion Science and Technology, Vol. 3, No. 1, 1971, pp. 1-16.

${ }^{16}$ Maslen, S. H., and Moore, F. K., "On Strong Transverse Waves Without Shocks in a Circular Cylinder," Journal of Aeronautical Science, Vol. 23, No. 6, 1956, pp. 583-593.

${ }^{17}$ Flandro, G. A., "Rotating Flows in Acoustically Unstable Rocket Motors," Ph.D. Thesis, Daniel and Florence Guggenheim Jet Propulsion Center, California Inst. of Technology, Pasadena, CA, 1967.

${ }^{18} \mathrm{Chu}, \mathrm{B} . \mathrm{T}$., "Analysis of a Self-Sustained Thermally Driven Nonlinear Vibration," Physics of Fluids, Vol. 6, No. 11, 1963, pp. 1638-1644.

${ }^{19} \mathrm{Chu}$, B.-T., and Ying, S. J., "Thermally Driven Nonlinear Oscillations in a Pipe with Traveling Shock Waves," Physics of Fluids, Vol. 6, No. 11, 1963, pp. 1625-1637.

${ }^{20}$ Sirignano, W. A., "A Theoretical Study of Nonlinear Combustion Instability: Longitudinal Mode," Ph.D. Thesis, Dept. of Aerospace and Mechanical Sciences, Princeton Univ, Princeton, NJ, 1964.

${ }^{21}$ Sirignano, W. A., and Crocco, L., "A Shock Wave Model of Unstable Rocket Combustors," AIAA Journal, Vol. 2, No. 7, 1964, pp. 1285-1296.

${ }^{22}$ Mitchell, C. E., Crocco, L., and Sirignano, W. A., "Nonlinear Longitudinal Instability in Rocket Motors with Concentrated Combustion," Combustion Science and Technology, Vol. 1, No. 4, 1969, pp. 269-274.

${ }^{23}$ Crocco, L., and Mitchell, C. E., "Nonlinear Periodic Oscillations in Rocket Motors with Distributed Combustion," Combustion Science and Technology, Vol. 1, No. 2, 1969, pp. 147-169.

${ }^{24}$ Zinn, B. T., "A Theoretical Study of Nonlinear Combustion Instability in Liquid-Propellant Rocket Engines," AIAA Journal, Vol. 6, No. 10, 1968. pp. 1966-1972.

${ }^{25}$ Hardesty, D. R., "Unsteady Combustion in Gaseous Propellant Rocket," Ph.D. Thesis, Dept. of Aerospace and Mechanical Sciences, Princeton Univ., Princeton, NJ; Air Force Office of Scientific Research Rept. AFOSR-TR-71-0922, June 1971.

${ }^{26}$ Zinn, B. T., and Lores, E. M., "Application of the Galerkin Method in the Solution of Nonlinear Axial Combustion Instabil ity Problems in Liquid Rockets," Combustion Science and Technology, Vol. 4, No. 6, 1972, pp. 269-278.

${ }^{27}$ Lores, E. M., and Zinn, B. T., "Nonlinear Longitudinal Instability in Rocket Motors," Combustion Science and Technology, Vol. 7, No. 6, 1973, pp. 245-256.

${ }^{28}$ Lovine, R. L., Dudley, D. P., and Waugh, R. C., "Standardized Stability Prediction Method for Solid Rocket Motors," Vols. I-III, Aerojet Solid Propulsion Co.; Air Force Rocket Propulsion Lab., Edwards AFB, CA, AFRPL-TR-76-32, 1976.

${ }^{29}$ Nickerson, G. R., Culick, F. E. C., and Dang, L. G., "Standard Stability Prediction Method for Solid Rocket Motors, Axial Mode Computer Program, User's Manual," Software and Engineering Associates, Inc.; Air Force Rocket Propulsion Lab., Edwards AFB, CA, AFRPL-TR-83-017, 1983.

${ }^{30}$ Levine, J. L., and Culick, F. E. C., "Numerical Analysis of Non! near Longitudinal Combustion Instability in Metalized Solid-Propellant kocket 
Motors," Vol. 1, Analysis and Results, Ultrasystems, Inc.; Air Force Rocket Propulsion Lab., Edwards, AFB, CA, AFRPL-72-88, 1972.

${ }^{31}$ Levine, J. L., and Culick, F. E. C., "Nonlinear Analysis of Solid Rocket Combustion Instability," Ultrasystems, Inc.; Air Force Rocket Propulsion Lab., Edwards AFB, CA, AFRPL-74-74-45, 1974.

${ }^{32}$ Levine, J. L., and Baum, J. D., "A Numerical Study of Nonlinear Instability Phenomena in Solid Rocket Motors," AIAA Journal, Vol. 21, No. 4, 1983, pp. 557-564.

${ }^{33}$ Awad, E., and Culick, F. E. C., "On the Existence and Stability of Limit Cycles for Longitudinal Acoustic Modes in a Combustion Chamber," Combustion Science and Technology, Vol. 46, No. 6, 1986, pp. 195-222.

${ }^{34}$ Yang, V., and Culick, F. E. C., "On the Existence and Stability of Limit Cycles for Traverse Acoustic Oscillations in a Cylindrical Combustion Chamber, I. Standing Modes," Combustion Science and Technology, Vol. 72 , No. 1,1990 , pp. 37-65.

${ }^{35}$ Yang, V., Kim, S. I., and Culick, F. E. C., “Triggering of Longitudinal Pressure Oscillations in Combustion Chambers, I: Nonlinear Gasdynamics," Combustion Science and Technology, Vol. 72, No. 5, 1990, pp. 183 214.

${ }^{36}$ Awad, E., "Nonlinear Acoustic Instability in Combustion Chambers," Ph.D. Thesis, Dept. of Mechanical Engineering, California Inst. of Technology, Pasadena, CA, 1983.

${ }^{37}$ Levine, J. L., and Baum, J. D., "Modeling of Nonlinear Combustion Instability in Solid Propellant Rocket Motors," Nineteenth Symposium (International) on Combustion, Combustion Inst., Pittsburgh, PA, 1982, pp. 769776.

${ }^{38}$ Yang, V., Kim, S. I., and Culick, F. E. C., "Third Order Nonlinear Acoustic Instabilities in Combustion Chambers, Part I: Longitudinal Modes," AIAA 25th Aerospace Sciences Meeting, AIAA Paper 87-0152, Jan. 1987.

${ }^{39} \mathrm{Kim}, \mathrm{S}$. I., "Nonlinear Combustion Instabilities in Combustion Chambers," Ph.D. Thesis, Mechanical Engineering, Pennsylvania State Univ., University Park, PA, 1989.

${ }^{40}$ Yang, V., Kim, S. I., and Culick, F. E. C., "Third Order Nonlinear Acoustic Instabilities in Combustion Chambers, Part II: Transverse Modes," AIAA Paper 88-0152, Jan. 1988.

${ }^{41}$ Flandro, G. A., "Energy Balance Analysis of Nonlinear Combustion Instability," Journal of Propulsion and Power, Vol. 1, No. 3, 1985, pp. 210221.

${ }^{42}$ Micheli, P. L., Lovine, R. L., Flandro, G. A., and Ditore, M., "Nonlinear Stability of Tactical Rocket Motors," Aerojet Solid Propulsion Co.; Air Force Rocket Propulsion Lab., Edwards AFB, CA, AFRPL-TR-84, 1984.

${ }^{43}$ Paparizos, L., and Culick, F. E. C., "The Two-Mode Approximation to Nonlinear Acoustics in Combustion Chambers. I. Exact Solution for Second Order Acoustics," Combustion Science and Technology, Vol. 65, No. 5,1989 , pp. 39-65.

${ }^{44}$ Paparizos, L., and Culick, F. E. C., "The Two-Mode Approximation to Nonlinear Acoustics in Combustion Chambers. II. Influence of Third-Order Acoustics and Mean Flow in Triggering," Combustion Science and Tech- nology (submitted for publication).

${ }^{45}$ Jahnke, C. C., and Culick, F. E. C., "An Application of Dynamical Systems Theory to Nonlinear Combustion Instabilities," AIAA Paper 93-0114, Jan. 1993.

${ }^{46}$ Doedel, E. J., and Kernevez, J. P., Software for Continuation Problems in Ordinary Differential Equations with Applications, Applied Mathematics Pub. 217-50, California Inst. of Technology, Pasadena, CA, 1984.

${ }^{47}$ Lord Rayleigh, "The Explanation of Certain Acoustical Phenomena," Royal Institution Proceedings, Vol. VIII, 1878, pp. 536-542; . also Lord Rayleigh, The Theory of Sound, Vol. II, Dover, New York, 1945, p. 226.

${ }^{48}$ Putnam, A. A., "General Considerations of Autonomous Combustion Oscillations," Non-Steady Flame Propagation, edited by G. F. Markstein, MacMillan, New York, 1964, Chap. F.

${ }^{49}$ Putnam, A. A., Combustion Driven Oscillations in Industry, American Elsevier, New York, 1971, Chap. 1.

${ }^{50}$ Zinn, B. T., "Pulsating Combustion," Advanced Combustion Methods, Academic Press, 1986, Chap. 5.

${ }^{51}$ Culick, F. E. C., "A Note on Rayleigh's Criterion," Combustion Science and Technology, Vol. 56, No. 5, 1987, pp. 159-166.

${ }^{52}$ Culick, F. E. C., "Combustion Instabilities and Rayleigh's Criterion," Modern Research Topics in Aerospace Propulsion (In Honor of Corrado Casci), edited by G. Angelino, L. De Luca, and W. A. Sirignano, SpringerVerlag, New York, 1992, pp. 135-151.

${ }^{53}$ Sterling, J. D., "Longitudinal Mode Combustion Instabilities in Air Breathing Engines," Ph.D. Thesis, California Inst. of Technology, Dept. of Mechanical Engineering, Pasadena, CA, 1987.

${ }^{54}$ Sterling, J. D., and Zukoski, E. E., "Longitudinal Mode Instabilities in a Dump Combustor," AIAA Paper 87-0220, Jan. 1987.

${ }^{55}$ Kantor, J. C., "A Dynamical Instability of Spark-Ignited Engines," Science, Vol. 224, June 1984, pp. 1233-1235.

${ }^{56}$ Daily, J. W., "Cycle-to-Cycle Variations: A Chaotic Process?" Combustion Science and Technology, Vol. 57, 1988, pp. 149-162.

${ }^{57}$ Keanini, R. G., Yu, K., and Daily, J., "A Strange Attractor in Ramjet Combustion," AIAA 27th Aerospace Sciences Meeting, AIAA Paper 896904, Jan. 1989.

${ }^{58}$ Sterling, J. D., "Characterization of the Nonlinear Dynamics of Experimental Signals: Distinguishing Between Noise and Chaos," Guggenheim Jet Propulsion Center, California Inst. of Technology, Documents on Active Control of Combustion Instabilities, Doc. Ci90-2, 1990.

${ }^{59}$ Sterling, J. D., "Characterization and Modeling of Aperiodic Pressure Oscillations in Combustion Chambers," AIAA Paper 91-2082, June 1991.

${ }^{60}$ Culick, F. E. C., Paparizos, L., Sterling, J., and Burnley, V., "Combustion Noise and Combustion Instabilities in Propulsion Systems," AGARD Conference Proceedings 512, Oct. 1991.

${ }^{61}$ Yang, Y., and Culick, F. E. C., "Stochastic Excitation of Acoustic Waves in a Combustion Chamber," 23rd JANNAF Combustion Meeting, 1986.

${ }^{62}$ Paparizos, L., and Culick, F. E. C., "Nonlinear Acoustics with Stochastic Sources," unpublished. 